Historic, Archive Document

Do not assume content reflects current scientific knowledge, policies, or practices. 
"Yours for Growing Satisfaction"

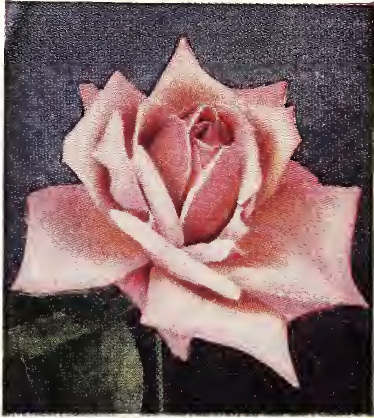

ROSE

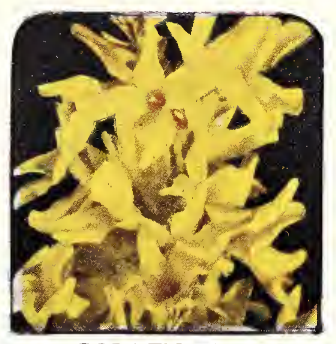

GOLDEN BELL

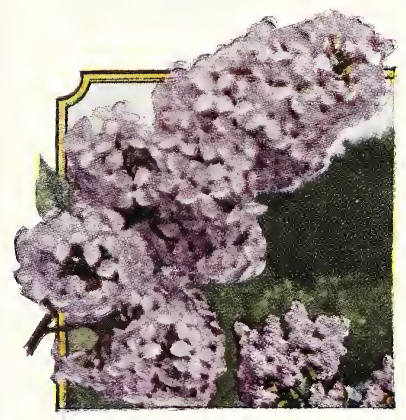

LILAC

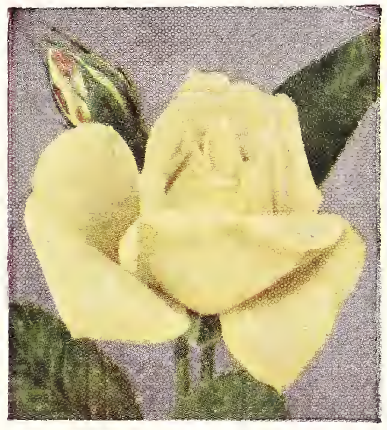

ROSE

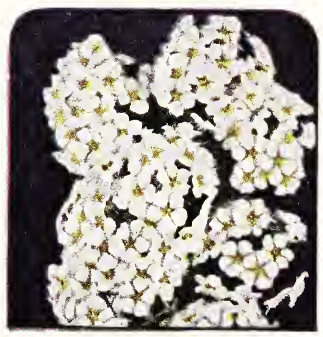

SPIREA V. $\mathrm{H}$.

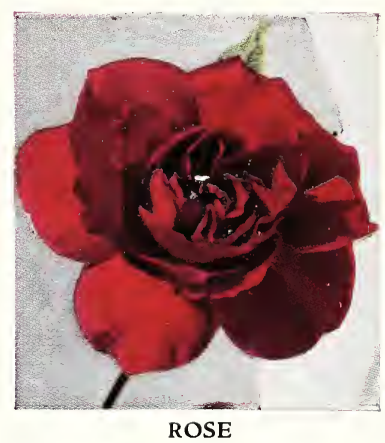

Beautiful Flowers, Handsome Trees, Shrubs, Evergreens and Luscious Fruits

are easily and inexpensively produced with Neosho Service and Products.

Our Customers say we chose the right motto"Yours for Growing Satisfaction"

Nurseries Co. , MISSOURI 


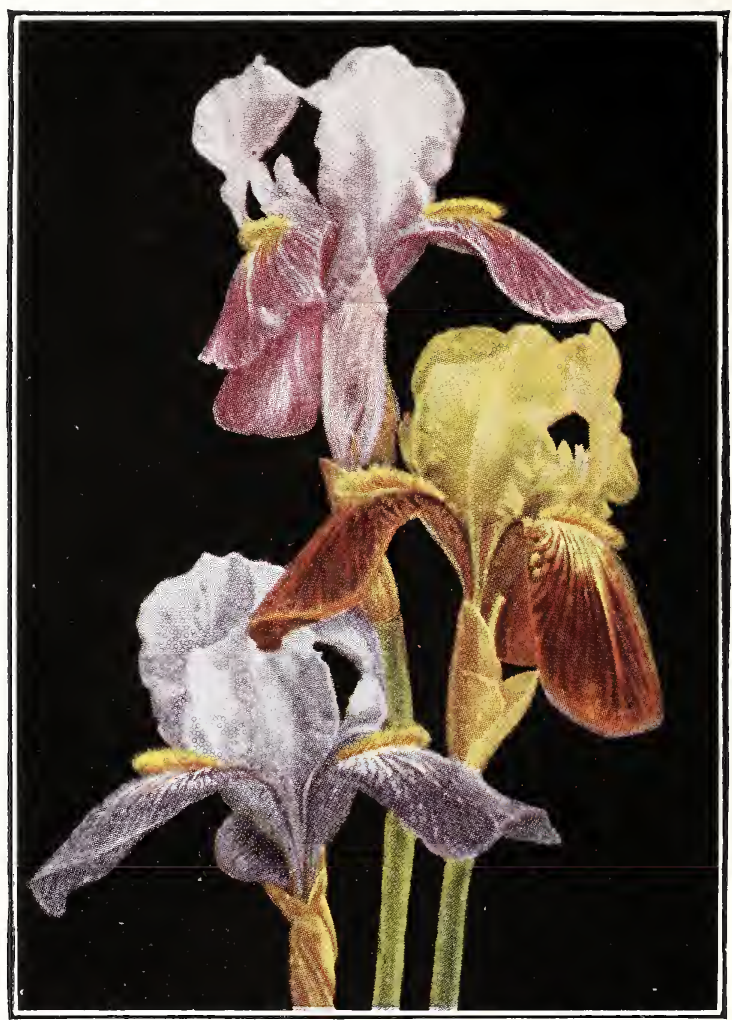

IRIS-(German) Iris germanica

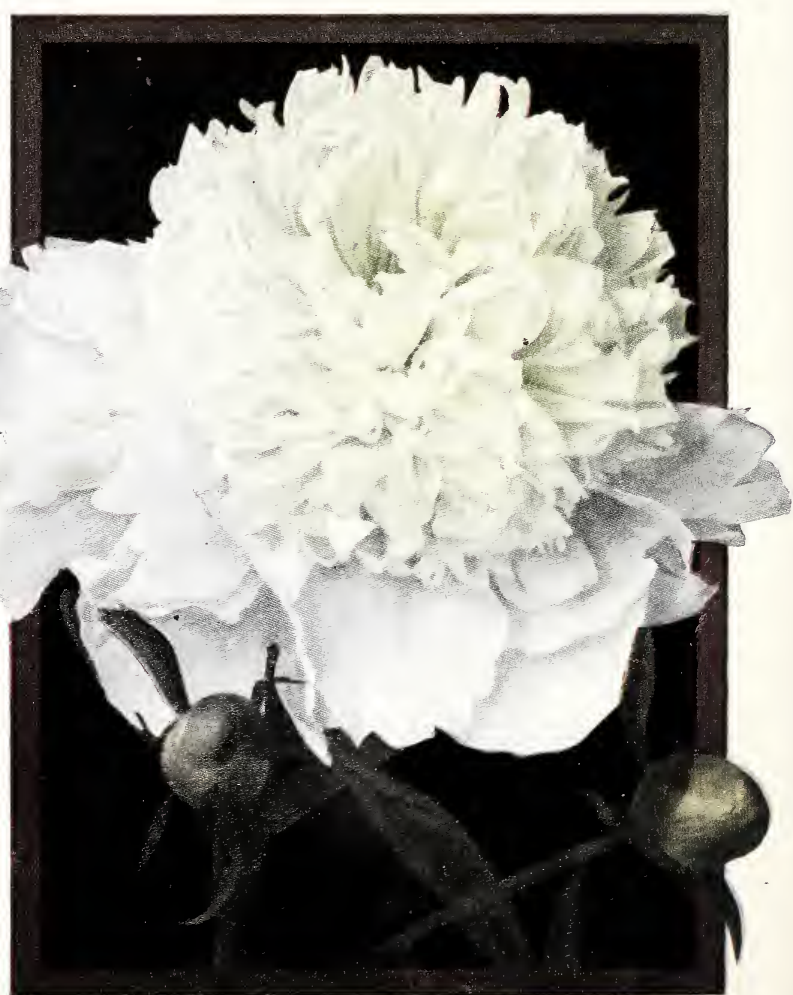

PEONY-Duke of Wellington 


\section{Better Trees and Plants}

Prices F. O. B. Neosho.

Subject to change without notice.

\section{INDEX TO CONTENTS}

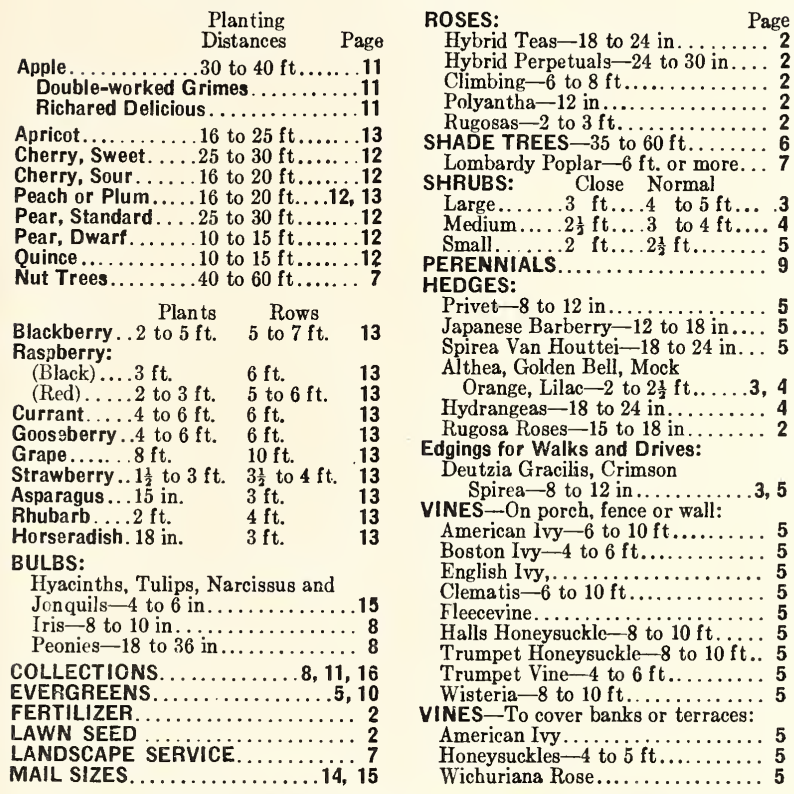

\section{EXPLANATION OF PRICES, ETC.}

TERMS: Cash on orders for less than $\$ 10.00$. (in orders for $\$ 10.00$ or more a deposit of 10 per cent is required with order, balance before shipment.

TRANSPORTATION CHARGES are payable by customer except where quoted prepaid by mail.

QUANTITY RATES: Apply on trees or plants of one class or kind. For instance, 25 apple or crabapple of one or more varieties entitles you to the 25 rate. But 5 apple, 5 peach, 5 plum, 5 pear and 5 cherry would take the 5 rate. Five shrubs of several varieties take the 5 rate.

INSPECTION: Every package bears the certificate of inspection of the State of Missouri. We welcome rigid official inspection such as is given in some states upon arrival.

GUARANTEE: We guarantee your satisfaction upon arrival of your order. If you should receive any unsatisfactory stock, heel it in subject to our disposal. All claims should be made immediately upon receipt of goods.

TRUE-TO-NAME WARRANTY: The best guarantee of trueness-toname is the precautions and care we take in handling and labeling our stock. If, however, any tree or plant should prove untrue-to-name, it is understood and agreed that the Neosho Nurseries shall be liable for the sum paid for the stock which may be proven untrue and shall not be liable for any greater amount.

\section{NOVELTY APPLE TREE FIVE VARIETIES}

We have a limited number of apple trees with from 4 to 5 different varieties budded on as many different branches.

These varieties ripen from early summer to late fall-Duchess, Wealthy, Grimes Golden, Jonathan and Delicious-all dependable, high grade apples.

These trees are 3 years old with trunks $\frac{11}{10}, 8 / 4$ and 1 inch thick.

PRICES-F. O. B. Neosho, cash with order. Satisfaction guaranteed upon arrival. Too large for Parcel Post.

5 Varieties on One Tree.......\$2.00 4 Varieties on One Tree...... $\$ 1.75$

"Yours for Growing Satisfaction",

NEOSHO NURSERIES CO.

NEOSHO, MO.

Our new Neosho Grower's Guide with 8 pages in full colors mailed free upon request. 


\section{ROSES-Prices are F. O. B. Neosho.}

Two-year field grown plants of first grade. $\star$ Everbloomers. The Climbers have 3 canes or more, but Dorothy Perkins, Excelsa and Wichuriana have 4 canes or more. Baby roses, 4 canes or more. Teas, Hybrid Teas and Hybrid Perpetuals, 3 canes or more, will give satisfactory blooms the first season.

One Neosho customer counted over 1,000 blooms the first year on 17 of our plants.

CLIMBING ROSES:

$\begin{array}{lcc}\text { Each } & 5 & 25 \\ \text { Rate } & \text { Rate } & \text { Rate }\end{array}$

American Pillar, bright crimson, white eye, yellow stamens. . . $\$ 0.75 \$ 0.65$

$\$ 0.55$

Climbing American Beauty, rosy crimson, long stems,

suitable for eut flowers..................... .60

Dr. Van Fleet, flesh pink ......................... .75

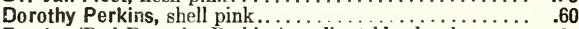

Excelsa (Red Dorothy Perkins), radiant blood red ...........60

*Flower of Fairfield, crimson, everblooming........... 1.00

Gardenia, bright yellow shading to cream............ .75

*Lady Ashtown, bright pink, everblooming................ 1.00

Mary Wallace, bright pink........................ $\quad .75$

Paul's Scarlet Climber, scarlet, shaded crimson......... 75

Silver Moon, silvery white, yellow stamens.......... $\quad .75$

Wichuriana, single white, trailing habit.............. $\quad .75$

$.50 \quad 45$

$.65 \quad .55$

$.50 \quad .45$

$.50 \quad .45$

$.85 \quad .75$

$.65 \quad .55$

$.85 \quad .75$

$.65 \quad .55$

$.65 \quad .55$

$.65 \quad .55$

$.65 \quad .55$

BABY ROSES-Dwarf Polyantha:

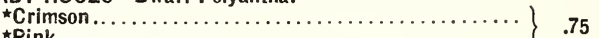

HYBRID PERPERTUALS:

Marshall P. Wilder, bright cherry-red...............
$\star$ Frau Karl Druschki (Snow Queen), purest white, large.

*Frau Karl Druschki (Snow Queen), purest white, large.

General Jacqueminot, rich crimson scarlet............

TEAS AND HYBRID TEAS:

*Gruss an Teplitz, bright crimson scarlet, very fragrant.

«Kaisarin Augusta Victoria, creamy white.............

* Killarney Pink, clear bright pink, hardy

* Lady Hillingdon, deep apricot yellow varying to orange.

* LaFrance, bright pink..

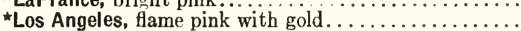

*Maman Cochet, pink.

* Madame Caroline Testout, light pink...............

«Radiance, pink.

*Red Radiance red............................

$\star W m$. R. Smith, silvery white and pink toned with

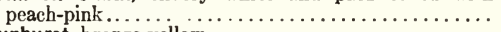

$\star$ Sunburst, bronze-yellow . . . . . . . . . . . . . . . . . .

*Souv. de Claudius Pernet, fadeless sunflower yellow...

*Souv. de Georges Pernet, brick red buds opening to terra-cotta pink.

*Madame E. Herriot, buds coral-red and orange, open-

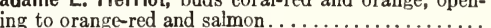

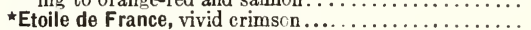

ॠD. L. Mock, deep carmine pink......................

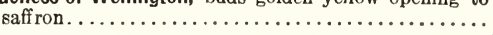

$\star$ Talisman, golden yellow and copper......................\$3.00 Each

Can ship these top grade plants by mail. Add 8 c per plant, $15 \mathrm{c}$ per 3 .

RUGOSA ROSES-Two-Year, No. 1:

The following Rugosa Roses and Hybrids are perfectly hardy, endure partial shade and are uscful for hedges or shrubs groups.

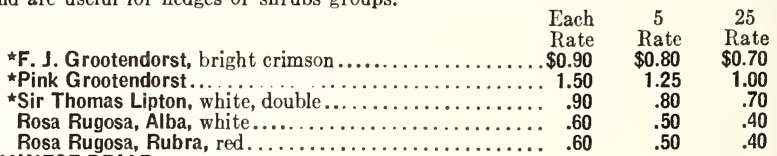

CHINESE BRIAR:

Hugonis ("Golden Rose of China"), deep gold to canary

flowers in April; a tractive shrub up to $6 \mathrm{ft}$. tall... \$0.

$\$ 0.80 \$ 0.70$

\section{LAWN SEED-Add 5c per lb. for postage.}

Our lawn seed are cleaned and recleaned and tested for germination. It pays to use good seed free from chaff and weed seed. On new lawns, use $1 \mathrm{lb}$. for 300 square feet; to replenish lawns, one-half as much.

\begin{tabular}{|c|c|c|c|c|}
\hline $\begin{array}{l}\text { STANDARD MIXTURE (Highest Grade). } 1 / 20.35 \\
\text { HOME GROUNDS MIXTURE..........20 } \\
\text { (For lawns that require annual reseeding. }\end{array}$ & $\begin{array}{l}1 \mathrm{lb} . \\
\$ 0.65 \\
.35\end{array}$ & $\begin{array}{r}2 \mathrm{lb} . \\
\$ 1.25 \\
.65\end{array}$ & $\begin{array}{l}3 \mathrm{lb} \\
\$ 1.75 \\
1.00\end{array}$ & $\begin{array}{r}51 b \\
\$ 2.75 \\
1.50\end{array}$ \\
\hline 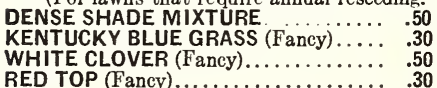 & $\begin{array}{l}.95 \\
.55 \\
.95 \\
.55\end{array}$ & $\begin{array}{l}1.80 \\
1.00 \\
1.80 \\
1.00\end{array}$ & $\begin{array}{l}2.60 \\
1.40 \\
2.60 \\
1.40\end{array}$ & \\
\hline
\end{tabular}

\section{FERTILIZER}

VIGORO-A valuable general fertilizer easily applied. Prices f. o. b. Neosho

$$
\begin{array}{lcc}
\text { Per 5 Lbs. Per 25 Lbs. } & \text { Per } 100 \text { Lbs. } \\
\$ 0.50 & \$ 1.75 & \$ 5.00
\end{array}
$$

The 5-lb. package can be sent by mail to points within 300 miles of Neosho for. 16c postage.

Quantities to Use-Cn lawns, 4 lbs. per 100 sq. ft. On shrubs, 4 lbs. per 100 sq. $\mathrm{ft}$. On hedges, 2 lbs. per $100 \mathrm{ft}$. of hedge. On trees, $3 \mathrm{lbs}$. for each inch of diameter of tree trunk, measuring $4 \mathrm{ft}$. frcm ground. Use in fall or spring on lawns: in spring and early summer for other plants. Directions for use are printed on each package. 
This list contains the best grades, well branched and well rooted.

(For smaller sizes see mail sizes, pages 14, 15)

The 5-Rate applies on 5 shrubs of one or more varieties.

Figures following names of varieties are heights when full grown.

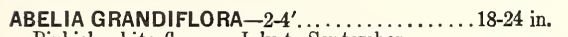

Pinkish white flowers, July to September.

$18-24$ in. $\quad \$ 1.00 \quad \$ 0.90 \quad \$ 0.80$

Specimens-Balled and Burlapped. ..... $\left\{\begin{array}{llll}30-36 & \text { in. } \quad 2.50 & 2.25 & \ldots .\end{array}\right.$

$\left\{\begin{array}{llll}24-30 \text { in. } & 1.80 & 1.60 & \ldots \\ 18-24 & \text { in. } & 1.50 & 1.35\end{array}\right.$

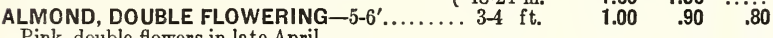

Pink, double flowers in late April.

AL THEA or ROSE OF SHARON $-8-12^{\prime} \ldots \ldots \ldots \ldots 3-4 \mathrm{ft} . \quad \begin{array}{lllll}.70 & .60 & .50\end{array}$

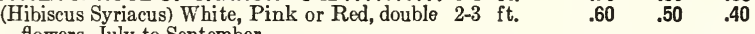

flowers, July to September.

$\begin{array}{llllllll}\text { ARROWWOOD (Viburnum Dentatum) }-8-12^{\prime} \ldots . . & 3-4 & \mathrm{ft} . & & .80 & .70 & .60\end{array}$

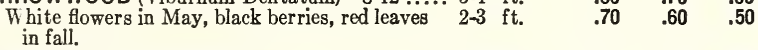

BARBERRY, JAPANESE- $-3-5^{\prime} \ldots \ldots \ldots \ldots \ldots \ldots 2-21 / 2 \mathrm{ft}$.

(Berberis Thunbergi)

Scarlet berries and leaves in fall.

BARBERRY, RED LEAVED $-2-4^{\prime} \ldots \ldots \ldots \ldots \ldots 15-18 \mathrm{in}$.

(Berberis Thunbergi A tropurpurea) $\cdots \cdots \cdots \cdots \cdots \cdots$

Red foliage all season. $9-12$ in.

BEAUTYBERRY (Callicarpa Purpurea) $-2-4^{\prime} \ldots \ldots 2-3 \mathrm{ft}$.

Violet berries in August.

BUTTERFLY BUSH (Buddleia Magnifica) -4-5' . . .2 Yr., No. 1

Lilac flowers, June-Sept.

COMMON NINE BARK-8-10'

(Physocarpus opulifolius)

White flowers, May.

$\begin{array}{lll}.50 & .40 \quad .35\end{array}$

$\begin{array}{lll}.40 \quad .30 & .25\end{array}$

$\begin{array}{lll}1.00 & .85 & .75\end{array}$

$\begin{array}{lll}.75 & .65 & .50 \\ .60 & .50 & .40\end{array}$

$\begin{array}{lll}.85 & .75 & .65\end{array}$

$\begin{array}{lll}.75 & .65 & .55\end{array}$

$\begin{array}{lll}.50 & .40 & .35\end{array}$

$\begin{array}{lll}.60 & .50 & .40\end{array}$

$\begin{array}{lll}.60 & .50 & .40 \\ .50 & .35\end{array}$

CORALBERRY (Indian Currant) $-3-5^{\prime} \ldots \ldots \ldots \ldots 2-3 \mathrm{ft}$.

(Symphoricarpus Vulgaris)

Red berries fall and winter.

C OTTONEASTER FRANCHETI- $-5-6^{\prime} \ldots \ldots \ldots \ldots 12-15 \mathrm{in.}$
Evergreen. Dark green leaves. Orange berries.

C RAPEMYRTLE (Lagerstroemia Indica)-10-25' . 2 2-3 ft,

Red, purple, pink or white. Fowers June to

August. Not hardy.

CUTLEAF STEPHANANDRA-5- $6^{\prime} \ldots \ldots \ldots \ldots 2-3 \mathrm{ft}$.

(Stephanandra flexnosa) Graceful, compact

shrub. Handsome foliage.

DESMODIUM PENDULIFLORUM-3-4'....... No. 1

(Bush Clover) (Lespedeza Formosa)

Rosy-purple flowers in September.

DEUTZIA GRACILIS-White- $2-3^{\prime} \ldots \ldots \ldots \ldots \ldots 18-24$ in.

White flowers in la te April.

DEUTZIA LEMOINEI-3-5' $\ldots \ldots \ldots \ldots \ldots \ldots .2-2 \frac{1}{2} \mathrm{ft}$.

White flowers in May.

DEUTZIA, PRIDE OF ROCHESTER-6-8'..... $3-4 \mathrm{ft}$.

White double flowers in May. $\quad 2-3 \mathrm{ft}$.

DOGWOOD, REDTWIGGED-6-10'.......... $3-4 \mathrm{ft}$.

(Cornus Alba Siberica)

Valuable for bright red bark.

DWARF BUSH HONEYSUCKLE- $3^{\prime} \ldots \ldots \ldots \ldots 18-24$ in.

(Diervilla trifida)

Yellow flowers, June.

DWARF BUTTERFLY BUSH--4-5'. ........... No. 1

(Buddleia Farquhar)

Lilac flowers, June-Sept.

ELDER GOLDEN-8-12'................. $3-4 \mathrm{ft}$.

(Sambucus Canadensis Aurea)

Yellow foliage all season.

$.50 \quad .40$

.35

$1.00 \quad .90 \quad .80$

$\begin{array}{lll}.85 & .75 & .65\end{array}$

$1.00 \quad .90 \quad .80$

$\begin{array}{lll}.85 & .75 & .65\end{array}$

$\begin{array}{lll}.60 & .50 & .40\end{array}$

$\begin{array}{lll}.50 & .40 & .35\end{array}$

$\begin{array}{lll}.60 & .50 & .40\end{array}$

$\begin{array}{lll}.50 & .40 & .35\end{array}$

$\begin{array}{lll}.60 & .50 & .40\end{array}$

$\begin{array}{lll}.50 & .40 & .35 \\ 60 & 50 & .40\end{array}$

$\begin{array}{lll}.60 & .50 & .40 \\ .50 & .40 & .35\end{array}$

$1.00 \quad .90 \quad .80$

$\begin{array}{lll}.65 & .55 & .45\end{array}$

$\begin{array}{lll}.75 & .65 & .55\end{array}$

$\begin{array}{lll}.75 & .65 & .55 \\ .65 & .55 & .45\end{array}$

$.65 \quad .55 \quad .45$

(Sorbaria sorbifolia) Fluffy heads of white flowers, June-July.

GLOBE FLOWER (Japanese Kerria) $-4-5$ '.

(Kerria Japonica flore-pleno)

Double golden yellow flowers, May-Sept.

$2-3 \mathrm{ft}$.

1.00

$3-4 \mathrm{ft}$.

GOLDEN BELL, Border-6-8'............

(Forsythia In termedia)

Yellow flowers in early April.

GOLDEN BELL, Weeping-4-6 $6^{\prime} . \ldots \ldots \ldots \ldots$

(Forsy thia Suspensa)

(Forsy thia Suspensa)
GOLDEN BELL, Fortunes-7-10'.........
(Forsythia Fortunei)

GOLDEN BELL, Greenstem-7-10'.......

(Forsy thia Virdissima)

GOLDEN CHAIN - 20-25',

(Laburnum Vulgare)

Yellow flowers in spring.

GOLDLEAF NINE BARK -8-10'

(Physocarpus opulifolius aurea)

Golden leaves, white flowers, May.

HERCULES CLUB - $18-20^{\prime} \ldots \ldots \ldots \ldots . .$.
(Devil's Walking Stick) (Aralia Spinosa)

White flowers, July. 


\section{SHRUBS-(Continued) Prices F. O. B. Neosho}

HIGH BUSH CRANBERRY-8-12' .......... 2-3 ft.

(Viburnum Opulus)

White flowers in May. Red berries in winter.

8-24 in.

Each $5 \quad 25$

Rate Rate Rate

$\begin{array}{rrr}.85 & .75 & .65\end{array}$

$.60 \quad .50$

Each $\quad 5 \quad 25$

Rate Rate Rate

$\begin{array}{llr}1.00 \quad .90 & .80\end{array}$

HONEYSUCKLE, EVERBLOOMING-15-20'.... 2 Yr.

(Lonicera heckrotti)

Flowers purple outside, yellow inside, July-Sept

HONEYSUCKLE, Japan Bush-6-8'..

(Lonicera Morrowi) White flowers in May, then red berries.

HONEYSUCKLE, TATARIAN, Pink-6-10'...

(Lonicera Tatarica) Pink flowers in late

$3-4 \mathrm{ft}$.

.60 April, then red berries.

HONEYSUCKLE, FRAGRANT $-6-8^{\prime} \ldots \ldots \ldots$.

(Lonicera Fragrantissima)

Dark green leaves, almost evergreen.

HYDRANGEA ARBORESCENS GRANDIFLO RA. $3-31 / 2 \mathrm{ft}$. (Hills of Snow) $-3-6^{\prime}$

White, double flowers, June to August.

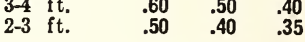

TYRANGEA PAN. GRANDIFLORA-6-10' ... 3-31/2 $\mathrm{ft}$.

(Bush Form) White flowers turning to pink, $2-3 \mathrm{ft}$.

then bronze, July to October.

HYDRANGEA PAN. GRANDIFLORA.......... 4-5 ft.

(Tree Form)

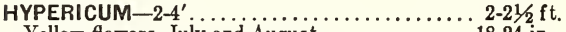

Yellow flowers, July and August.

JAPAN OUINCE (Cydonia Japonica) $-3-5^{\prime} \ldots \ldots \ldots 2-3 \mathrm{ft}$.

Scarlet flowers, early April. $18-24$ in.

JETBEAD or WHITE KERRIA-3-5' ......... 3-4 ft.

(Rhodotypus Kerroides) 2-3 ft.

White flowers May, black berries. $\quad 18-24$ in

LILAC-BUDDED FRENCH (Bush Form)-6-10'. . 3-4 ft.

(Syringa Hybrid)

$2-3 \mathrm{ft}$.

Dark purplish red, double white, or double blue.

Large flowers in late April.

LILAC-CHINESE-8-10'............... 3-4 ft.

(Syringa Climensis Rothomogensis) $\quad 2-3 \mathrm{ft}$

Reddish purple, early May, free bloomer, hardy.

LILAC-COMMON PURPLE-8-15'.......... $3-4 \mathrm{ft}$

(Syringa Vulgaris)

2-3 $\mathrm{ft}$.

Flowers early spring.

LILAC-PERSIAN PURPLE-6-10 $0^{\prime} \ldots \ldots \ldots \ldots 3-4 \mathrm{ft}$.

(Syringa Persica) Free bloomer, early May. $\quad 2-3 \mathrm{ft}$.

MOCK ORANGE-Sweet-8-12'............. 3-4 ft.

(Philadelphus Coronarius)

Fragrant white flowers in May.

MOCK ORANGE, LEMOINES $-4-6^{\prime} \ldots \ldots \ldots \ldots .21 / 2-3 \mathrm{ft}$.

(Philadelphus Lemoinei)

Fragrant white flowers in May. 18-24 in.

MOCK ORANGE, VIRGINALIS-6-8' $\ldots \ldots \ldots, 3-4 \mathrm{ft}$

(Philadelphus Virginalis) $2-3 \mathrm{ft}$.

Large white, semi-double, fragrant flowers in 18-24 in. May-June,

MOCK ORANGE, GOLDEN-Dwarf-3-5' .....18-24 in

(Philadelphus Aurea) Yellow leaves all season.

MOCK ORANGE, WHITE BOUOUET $-4-5^{\prime} \ldots \ldots$ 18-24 in

(Philadelphus boquet blanc) White flowers, May.

PEARL BUSH $-6-10^{\prime} \ldots \ldots \ldots \ldots \ldots \ldots \ldots \ldots 2-3 \mathrm{ft}$.

(Exochorda grandiflora) White flowers, May.

PLUM, PURPLE LEAVED-10-15' $\ldots \ldots \ldots \ldots .4-5 \mathrm{ft}$.

(Prunus Pissardi) Purple leaves all season. $3-4 \mathrm{ft}$.

PLUM, DOUBLE FLOWERING-6-10'....... 4-5 ft.

(Prunus Triloba) Double pink flowers, iate April. $3-4 \mathrm{ft}$.

PRIVET, REGELS $-4-6^{\prime} \ldots \ldots \ldots \ldots \ldots \ldots \ldots 2-21 / 2 \mathrm{ft}$

Ligustrum Regelianuma)

White flowers, black berries.

ROSA HUGONIS (Golden Rose of China) $-4-6$ ' . . 2 Yr., No. Golden yellow flowers, April.

ROSA RUGOSA, White (Alba), Red (Rubra)-3-5'. 2 Yr., No. 1

Flowers in May.

Double crimson flowers, all season.

Sir Thomas Lipton-4-6' ................2 Yr., No. $1 \quad .90$

Double white flowers, June and occasionally all summer.

SILVERBELL-12 $15^{\prime}$

(Halesia tetraptera) White bell-shaped flowers,

May.

SHRUBBY CINQUEFOIL-2-4' $\ldots \ldots \ldots \ldots \ldots 15-18$ in.

(Potentilla fruiticosa) Yellow flowers all summer.

SNOWBALL (Viburnum Opulus Sterile) $-8-12^{\prime} \ldots 3-4 \mathrm{ft}$.

Large clusters white flowers in May. $\quad 2-3 \mathrm{ft}$.

SNOWBERRY (Symphoricarpus Racemosus) -3-5'. $2-3 \mathrm{ft}$.

White berries, June into winter.

SPIREA, THUNBERG'S (S. Thunbergii)-3-5' . . 2-21/2 ft.

White flowers, April, fea thery foliage. 18-24 in.

SPIREA CRIMSON (S. Anthony Waterer)-2-3'... 2-21/2 $\mathrm{ft}$.

Bright red flowers all summer. $18-24$ in.

SPIREA, BILLARD'S (S. Billardi Rosea) $-1-6^{\prime} \ldots 3-4 \mathrm{ft}$.

Pink flowers all summer.

$\begin{array}{rrr}.90 & .75 & .60 \\ .60 & .50 & .40 \\ .50 & .40 & .35 \\ 1.00 & .80 & .70 \\ .80 & .70 & .60 \\ .60 & .50 & .40 \\ 2.00 & 1.75 & 1.50 \\ 1.50 & 1.25 & 1.00 \\ 1.00 & .80 & .70 \\ .90 & .70 & .60 \\ .75 & .65 & .55 \\ .60 & .50 & .40 \\ 1.00 & .80 & .70 \\ .80 & .70 & .60 \\ .60 & .50 & .40 \\ 1.75 & 1.50 & 1.25 \\ 1.50 & 1.25 & 1.00\end{array}$

$\begin{array}{lll}.90 & .80 & .70\end{array}$

$\begin{array}{lll}.75 & .65 & .55\end{array}$

$\begin{array}{lll}.80 & .70 \quad .60\end{array}$

$\begin{array}{lll}.60 & .50 & .40\end{array}$

$\begin{array}{lll}.90 & .80 & .70\end{array}$

$\begin{array}{lll}.75 & .65 & .55\end{array}$

$.60 \quad .50 \quad .40$

$\begin{array}{lll}.75 & .65 & .55\end{array}$

$\begin{array}{lll}.60 & .50 & .40 \\ .40 & .30 & .25\end{array}$

$\begin{array}{lll}1.00 & .90 & .80\end{array}$

$\begin{array}{lll}.80 & .70 & .60\end{array}$

$\begin{array}{lll}.60 & .50 \quad .40\end{array}$

$1.00 \quad .90 \quad .80$

$\begin{array}{lll}.65 & .55 & .45\end{array}$

$\begin{array}{lll}.65 & .55 \quad .45\end{array}$

$\begin{array}{lll}1.25 & 1.10 & 1.00\end{array}$

$\begin{array}{lll}1.00 & .90 & .80\end{array}$

$\begin{array}{lll}1.75 & 1.50 & 1.25\end{array}$

$\begin{array}{lll}1.50 & 1.25 & 1.00\end{array}$

$.40 \quad .35 \quad .30$

$.90 \quad .80 \quad .70$

$\begin{array}{lll}.60 & .50 & .40\end{array}$

1.251 .00

$.80 \quad .70$

$\begin{array}{lll}1.75 & 1.50 & 1.25\end{array}$

$\begin{array}{lll}.75 & .60 & .50\end{array}$

$\begin{array}{lll}1.25 & 1.10 & 1.00\end{array}$

$\begin{array}{lll}1.00 & .90 & .80\end{array}$

$\begin{array}{lll}.75 & .60 & .50\end{array}$

$\begin{array}{lll}.50 & .40 & .35\end{array}$ 
SPIREA, BRIDAL WREATH (S. Prunifolia)-6-8'. 3-4 ft. Double white flowers, early April. $2-3 \mathrm{ft}$. SPIREA, DWARF WHITE (S. Callosa Alba)-2-3'.18-24 in. White flowers all summer.

SPIREA, DWARF PINK (S. Callosa Rosea)-2-3'..18-24 in. Light pink flowers in summer.

SPIREA, FROEBEL'S (S. Froebeli)-2-4' $\ldots \ldots \ldots 2-21 / 2 \mathrm{ft}$.

Bright crimson flowers in summer.

SPIREA, VAN HOUTTE'S (S. Van Houttei)-6-8'. $3-4 \mathrm{ft}$. White flowers late April. $2-3 \mathrm{ft}$. SUMAC, STAGHORN (Rhus Typhina)-15-25'... 3-4 $\mathrm{ft}$. Red fruits late summer. Leaves yellow and purple in fall.

SWEET PEPPER BUSH (Clethra Alnifolia)-3-5'..18-24 in. White fragrant flowers, July-Sept.

SUMAC FRAGRANT-2-2',................ (Rhus Canadensis Aromatica) $\cdots \cdots \cdots \cdots \cdots \cdots \cdots \cdots \cdots \cdots$ Red foliage and berries in fall. $\quad 4-5 \mathrm{ft}$. SUMAC-Smooth (Rhus Glabra)-8-12'...... ${ }^{3-4} \mathrm{ft}$. Fern-like foliage, red in fall. Crimson berries. ${ }_{2-3} \mathrm{ft}$.

TAMARISK AFRICANA-8-12,.............. $3-4 \mathrm{ft}$.

Pink flowers in May, fea thery foliage. $\quad 2-3 \mathrm{ft}$.

TAMARIX HISPIDA-8-12' ............. 4-5 ft. Feathery silver foliage, bright pink flowers, June. $3-4 \mathrm{ft}$. WEIGELA-Pink (Weigela Rosea)-6-8' $\ldots \ldots \ldots \ldots 3-4 \mathrm{ft}$. Rose pink flowers, May.

WEIGELA HYBRID, Red-3-5' ........... 3-4 $\mathrm{ft}$. (Weigela Eva Rathke) Crimson flowers, June. ${ }_{2-3} \mathrm{ft}$. WHITE FRINGE-20-25'.................. (Chionanthus Virginica)

Lace-like white flowers, May.

WINGED EUONYMUS $-8-10^{\prime} \ldots \ldots \ldots \ldots \ldots .2-3 \mathrm{ft}$.

(Euonymus Alatus) Corky winged bark, crimson leaves in fall.

WINTERBERRY $-6-8^{\prime} \ldots \ldots \ldots \ldots \ldots \ldots \ldots 21 / 2-3 \mathrm{ft}$.

(Ilex Verticilla ta) Red berries into winter.

Each $5 \quad 25$

$\begin{array}{rrr}\text { Rate } & \text { Rate } & \text { Rate } \\ .75 & .60 & .50\end{array}$

$\begin{array}{lll}.60 & .50 & .50\end{array}$

$\begin{array}{lll}.60 & .50 & .40\end{array}$

$\begin{array}{lll}.60 & .50 \quad .40\end{array}$

$\begin{array}{lll}.60 & .50 & .40\end{array}$

$\begin{array}{lll}.50 & .40 & .35 \\ .50 & .40 & .35\end{array}$

$.50 \quad .40 \quad .35$

$\begin{array}{lll}.40 & .30 & .25 \\ .65 & .55 & .45\end{array}$

$1.00 \quad .90 \quad .80$

$1.00 \quad .90 \quad .80$

$\begin{array}{lll}.80 & .70 & .60\end{array}$

$.75 \quad .65 \quad .55$

$\begin{array}{lll}.60 & .50 & .40\end{array}$

$\begin{array}{lll}.50 & .40 & .35 \\ .60 & 50 & 50\end{array}$

$\begin{array}{lll}.50 & .40 & .35\end{array}$

$\begin{array}{lll}1.00 & .90 & .80\end{array}$

$\begin{array}{lll}.85 & .75 & .65\end{array}$

$\begin{array}{lll}.60 & .50 & .40\end{array}$

$\begin{array}{lll}.50 & .40 & .35\end{array}$

$\begin{array}{lll}.85 & .75 & .65\end{array}$

$\begin{array}{lll}1.75 & 1.50 & 1.25\end{array}$

$\begin{array}{lll}1.25 & 1.10 & 1.00\end{array}$

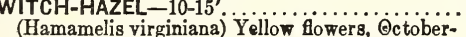

November.

$\begin{array}{lll}1.50 & 1.35 & 1.10\end{array}$

$\begin{array}{lll}1.50 & 1.35 & 1.20\end{array}$

\section{HEDGE PLANTS-Prices F. O. B. Neosho}

Hedge plants with many canes are worth more and give quicker and better results. We offer the best.

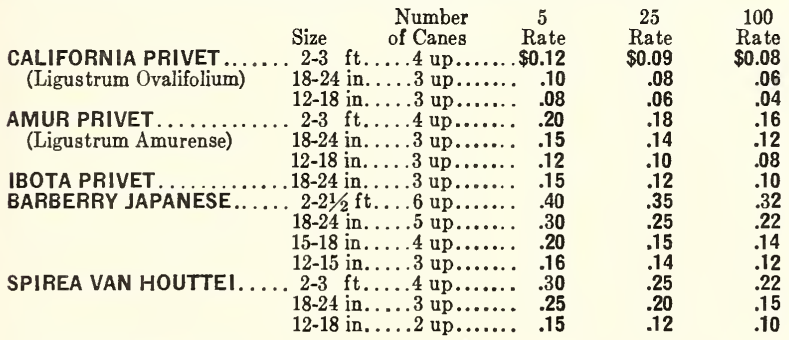

\section{VINES-2 Yr., No. 1}

Add for postage: $8 c$ for 1 vine, $25 c$ for 5 vines.

\section{SCARLET TRUMPET HONEYSUCKLE (Lonicera}

Sempervirens), Scarlet.....................15 to $20 \mathrm{ft}$.

RUMPET VINE (Bignonia Radicans), Scarlet and

Orange .............................20 to $30 \mathrm{ft}$.

WISTERIA JAPANESE (Grafted), Blue.............

WISTERIA (Seedlings).....................

CHINA FLEECEVINE (Polygonum Auberti), White...25 to $30 \mathrm{ft}$.

\section{BROADLEAVED EVERGREENS}

\section{Evergreen Shrubs-Prices F. O. B. Neosho} (Balled and Burlapped)

\section{AMERICAN HOLLY........................... $\quad \$ 12.00$ Each}

(Ilex Opaca)

Glossy leaves, scarlet berries in winter. Enjoys

partial shade.

OREGON HOLLY GRAPE . . . . . . . . . . . . . .

(Mahonia Aquifolia)

Green leaves turn scarlet in fall. Blue berries.

Grows 4 to $5 \mathrm{ft}$., enjoys partial shade.

Prices on Azalea, Rhododendron, Boxwood, Mountain Laurel upon request. 


\section{SHADE AND ORNAMENTAL TREES}

\section{Prices are F. O. B. Neosho.}

ASH, GREEN (Grows 50 to $60 \mathrm{ft}$ ) . . . . . Size

Fraxinus Viridis)

Round-topped head. Moderately rapid grower. $\quad 6-8 \mathrm{ft}$.

Fraxinus Americana) Tall, fairly rapid growing, .....

broad-headed. Purple leaves in fall.

(Sorbus Aucuparia)

(Valuable for orange-scarlet berries, Aug. to fall.)

BIRCH, WHITE (Grows 30 to $50 \mathrm{ft}$.) ............6. 6-7 ft. (Betula Alba)

BIRCH, CUTLEAF WEEPING (Grows 30 to $40 \mathrm{ft}$.) . . . 6-7 ft. (Betula Laciniata)

White bark, drooping branches.

BOX ELDER (Ash-leaved Maple) (Grows 30 to $60 \mathrm{ft}$.)... 6-8 ft. $\quad 1.25$ (Acer Negundo) Rapid growing, endures drought.

CATALPA, UMBRELLA-2-yr. heads, 5 -ft. stems $\ldots \ldots \ldots \ldots \ldots \ldots 2.00$

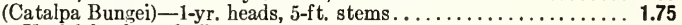
Useful for formal effects.

CATALPA, WESTERN (Grows 30 to $35 \mathrm{ft}$.) ..........6-8 $\mathrm{ft}$ (Catalpa Speciosa) White flowers, May. Rapid growing.

CHERRY, JAPANESE FLOWERING (Balled and Burlapped) (Prunus J. H. Veitch)............. 3-4 ft. Double rose flowers in May. Grows 30 to $40 \mathrm{ft}$

CRAB, BECHTEL DOUBLE FLOWERING (Grows 10 to $15 \mathrm{ft}$. (Pyrus Ioensis) $\ldots \ldots \ldots \ldots \ldots \ldots \ldots \ldots \ldots \ldots \ldots+4-5 \mathrm{ft}$.

CRAB, RED FLOWERING (Grows 15 to $20 \mathrm{ft}$ ) $\ldots \ldots .4-5 \mathrm{ft}$

(Malus Hopa) Rose colored flowers, late April. Red fruit in fall. Very hardy.

CRAB, PARKMAN (Grows 10 to $15 \mathrm{ft}$.).......... 4-5 ft.

(Malus Halliana Parkmani) Bright rose flowers, early May.

CRAB, FLORIBUNDA (Grows 10 to $15 \mathrm{ft}$.) ........ 4-5 ft.

(Malus Floribunda) Bright pink flowers, early May. Yellow fruits.

DOGWOOD, WHITE FLOWERING (Grows 15 to $25 \mathrm{ft}$ ) $5-6 \mathrm{ft}$.

(Cornus Florida) Large white flowers, April. Leaves crimson in fall. $3-4 \mathrm{ft}$.

DOGWOOD, RED FLOWERING (Grows 15 to $20 \mathrm{ft}$.) ... 2-3 ft. (Cornus Florida Rubra) Bright pink flowers, April

ELM, AMERICAN (Grows 80 to $100 \mathrm{ft}$.) ..........10-12 ft. (Ulmus Americana) Fairly rapid growing. $8-10 \mathrm{ft}$

$\begin{array}{ll}8-10 & \mathrm{ft} . \\ 6-8 \mathrm{ft} .\end{array}$

(Ulmus Americana Moline) Compact, fast growing, large leaves.

ELM, SIBERIAN OR CHINESE. ........... 8-10 ft.

(Ulmus Pumila) Leaves smaller, branches more slender 6-8 ft. than American. Very fast grower, very hardy, $\quad 5-6 \mathrm{ft}$. endures drought.

HOLLY, AMERICAN (Balled and Burlapped) . .......4-5 ft.

(Ilex Opaca) Evergreen leaves, smooth and spiny Red berries. A shrub or small tree.

LINDEN, AMERICAN (Grows 60 to $90 \mathrm{ft}$.) .........6-8 $\mathrm{ft}$.

(Tilia Americana) Rapid grower, large glossy leaves, fragrant yellow flowers, June.

MAGNOLIA, SOULANGE'S (Balled and Burlapped)

(Grows 15 to $18 \mathrm{ft}$.) (Magnolia Soulangeana)........ 3-4 ft. Large purplish-pink and white flowers, late April. Hardy, bush form

MAGNOLIA, SWEET BAY (Balled and Burlapped)

(Grows 15 to $18 \mathrm{ft}$.) (Magnolia Glauca)............ 3-4 ft. Glossy leaves, nearly evergreen. White flowers, April.

MAPLE, SCHWEDLER'S PURPLE LEAF

$$
\text { (Grows } 40 \text { to } 60 \mathrm{ft} \text {.). 6-7 ft. }
$$

4.00

(Acer Platanoides Schwedleri) Purplish-crimson leaves in spring turning to dark green.

MAPLE, NORWAY (Grows 50 to $60 \mathrm{ft}$.) . . . . . . . 6-8 $\mathrm{ft}$

(Acer Platanoides)

Handsome round-headed tree. Leaves dark green.

Yellow in fall. Fine for lawn or street planting.

MAPLE, SILVER (Grows 60 to $80 \mathrm{ft}$.) . . . . . .

(Acer Dasycarpum)

Very fast grower. Valuable for quick shade.

$2.50-2.00$

MAPLE, SUGAR (Grows 50 to $75 \mathrm{ft}$.) $\begin{array}{lrr}.13 / 4-2 \text { in. } \quad 10.00 & 8.00 \\ 8-10 \mathrm{ft} & 4.50 & 3.60\end{array}$

(Acer Saccharum) Yellow and red fall foliage. $\quad 8-10 \mathrm{ft}$. Slow growing. Long lived.

(Morus Tatarica) Useful for low windbreaks or hedges. Birds, poultry and hogs are fond of the fruit. 


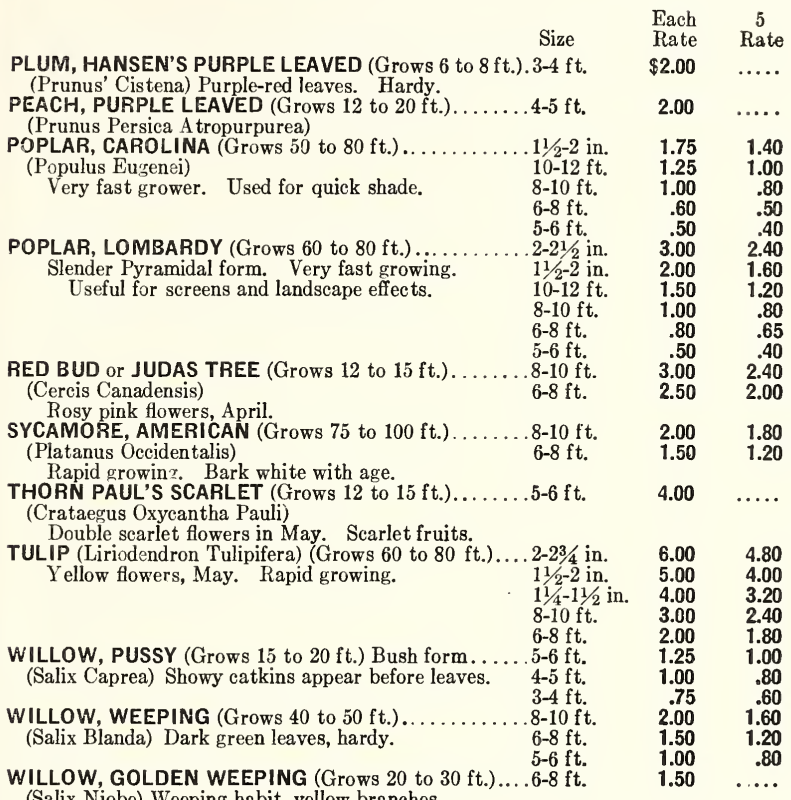

\section{NUT TREES-Prices F. O. B. Neosho.}

BLACK WALNUT-Ohio, Thomas-The kernels usually erack out in halves. Quality is excellent. Thrive in nearly every state...4-6 ft. $\quad \$ 3.00$ ENGLISH WALNUT-Franquette-A large ornamental tree with fine flavored thin shell nuts...........................

PECAN-Northern varieties, Butterick, Indiana-Adapted for Ozarks of Missouri and North. Thin shell, fine quality.......4-6 ft. PECAN - Southern varieties, Stuart, Moneymaker...................

\section{Neosho Landiscape Service}

The attractiveness, the comfort and the value of a home can be greatly increased by the proper use of plant materials.

You can secure such desirable results by sending us the information asked for below with your check for $\$ 5.00$. This sum will be applied on the purchase price of plant materials which you order from us. We will submit for your approval a plan and list of the plants required with an estimate of their cost.

Give all dimensions of building and lot.

Show location of buildings on lot.

Give points of compass.

Show location of all trees and shrubbery on place.

Show location of all walks, drives, etc.

Give all grades, show terraces, etc.

Show any objectionable views to be screened.

Attach kodak views, taken from north, south, east and west sides of lot, if possible.

Show where windows in first floor are placed.

Is the house one, two or three stories?

Height of foundation and windows.

Do you like a lot of shrubbery?

Do you want any hedges?

Where preferred?

Do you want us to locate trees, drives and walks?

Do you prefer any particular kind of trees?

Do you want a rose bed?

A formal flower garden?

Fruit trees?

Evergreens?

A vegetable garden?

Whore preferred?

What is the character of soil?

State how much you are willing to spend, also if you wish to plant part this season and the balance next season. 


\section{IRIS-Prepaid Prices.}

These exquisite flowers are easily grown. They pay big dividends in any garden. Be careful not to plant them too deep. The crown should be covered only 2 inches deep.

The figure following the name of the variety is the rating by the American Iris Society. Heights of the stalks are given in inches.

GERMANICA VARIETIES - Named in order of blooming.

$$
\text { Per } 3 \text { Per } 12 \text { Per } 25
$$

Black Prince (78) 24 in. Dark purplish blue; blooms nearly 30 days, beginning almost a month ahead of Orientalis.

Perfection (78) 30 in. Purple and lavender, very beau tiful.

San Souci (40) 24 in. Canary yellow and crimson brown.

Madame Chereau (74) 30 in. White frilled

SIBERIAN

$$
\begin{aligned}
& \text { with clear blue. } \\
& \begin{array}{c}
\text { Orientalls-18 in. Intense blue. Narrow } \\
\text { leaves. }
\end{array}
\end{aligned}
$$

GERMANICA-PRIZE WINNERS

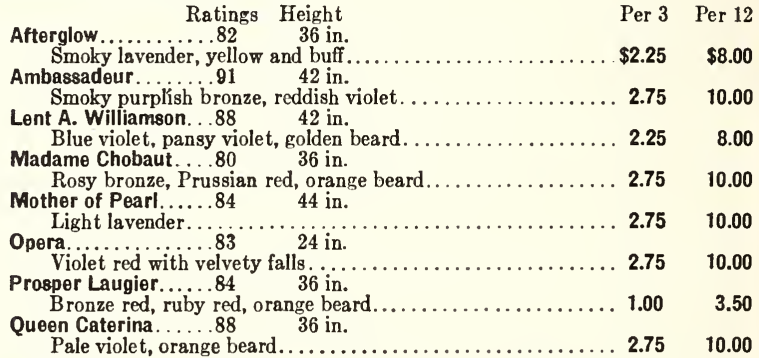

JAPANESE IRIS (Iris Kaempferi)-Flowers are rather flat and wide, appearing after the German varieties are through. Stalks are 2 to $3 \mathrm{ft}$. tall.

Gekka-no-naml-Glistening white. (Waves under Moonlight.)

Koko-no-Iro-Purple petals, yellow center. (Purple and gold.)

Moniji-no-takl-Rosy crimson, fea thered in white, double. (Maple Waterfall.)

Uchlu-Cerulean blue, golden center. (Universe.)

IRIS-Germanica..........................12 for $\$ 1.00$ postpaid

Three each of four colors. Our selection.

IRIS-Siberian.............................15 for $\$ 1.00$ postpaid

\section{PEONIES-Prices F. O. B. Neosho.}

The most widely planted and popular of all hardy flowers. Easy to grow, all they ask is good soil and winter mulch. Plant only 2 to 3 in. deep.

Double Blossoms-Strong Divisions, 3 to 5 Eyes.

WHITES

Each Rate 5 Rate

Couronne d'Or-White with gold stamens and carmine tips.

$$
\text { Late...................................\$0.50 \$0.45 }
$$

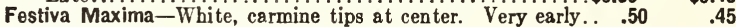

Duchess de Nemours - White, green tips at center. Fragrant. Midseason..................................50 .45

Duc De Wellington-Ivory white, large, double, fragrant.

Midseason..................................... 50

PINKS

Eugene Verdier-Pink, large, late................... $1.00 \quad .85$

Edulis Superba-Bright rose pink, very early ................ $\quad .75 \quad .65$ REDS

Duchess De Orleans-Deep pink, midseason................. $\quad .75 \quad .65$

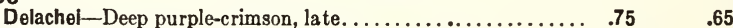

Meissonier-Brilliant reddish purple, midseason..............75 $\quad .75$

Nigricans-Dark crimson, compact bloom, midseason.......... $.75 \quad .65$

Prince Imperial-Brilliant purplish scarlet, very large, la te... $\quad .75 \quad .65$

Officinalis Rubra-Deep red, earliest of all Peonies............. $1.25 \quad 1.00$

Felix Crousse-Ruby red, late midseason ................ $1.00 \quad .85$

RARE VARIETIES

Walter Faxon-Bright pink, deepening toward center, midseason....\$7.50 each

Richard Carvel-Bright crimson, early .......................6.00 each

Mme. Jules Dessert-Creamy white with buff and salmon shades,

midseason .............. 6.00 each

Peony Collection...............\$2.00 Postpaid 3 white, 1 pink, 1 red. Our selection of named varieties.

3 white peonies, our selection, $\$ 1.00$ Postpaid. 


\section{PERENNIALS-Postpaid.}

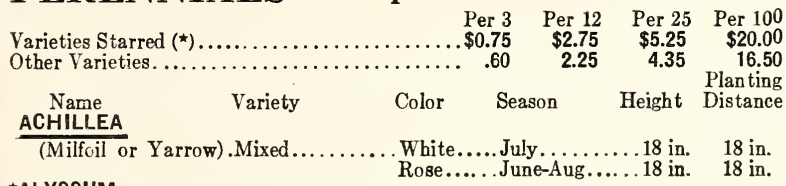

\section{^ALYSSUM}

Rose.... June-Aug 18 in 18 in

(Rock Madwort). . Saxatile CcmpactumYellow....April-May .....10 in. 6 in. ASTERS

Michaelmas Daisy.Feltham Blue......Blue......August.......36 in. 24 in.

Novae Angliae....Roseum Superbum.Red........August..........36 in. 24 in.

BABY'S BREATH

(Gypsc phila)......Paniculata.........White.....July-Sept....24-36 in. 12 in. BALLOON FLOWER

(Pla tycodon)...... Grandiflorum...... Blue...... June-Oet...12-36 in. 15 in ^BELLFLOWER

(Campanula) ...... Carpatica. ........ Blue..... June-Oct..... 8 in. 6 in.

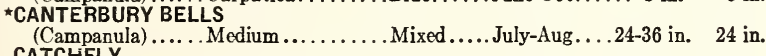

CATCHFLY

(Lychnis) ........ . Viscaria Splendans. Rosy-red. . June-July....18-24 in. 15 in.

CHRYSANTHEMUM
(Double)

COLUMBINE

$\left.\begin{array}{r}\text { (Aquilegia)....... Caerulea......... Blue..... } \\ \text { Chrysantha......... Yellow... } \\ \text { Mixed............ Various. }\end{array}\right\}$ May-June....24 in. $20 \mathrm{in.}$

CORNFLOWER ASTER

(Stokesia) .

DELPHINIUNM

(Larkspur)....... Belladonna..........t. Blue. )

Bellamosa.........Dk. Blue $\}$ June-Oct.....48 in. 30 in

ENGLISH DAISY

(Bellis) ......... Perennis. . . . . White..... April-June....3-6 in. 6 in.

*FALSE INDigo

(Baptisia) ......Australis..........Blue.....June........ 3-5 ft. $20 \mathrm{in.}$

*FORGET-ME-NOT

(Myosotis) .......Palustris.........Blue......Summer...... 8 in. 6 in.

^FOX GLOVE

(Digitalis) ........Giant Hybrids..... Mixed.....June-July.....36 in. 30 in.

GAILLARDIA

(Blanket Flower) ...............Red \& Yel. Summer......24 in. $12 \mathrm{in.}$

GOLDEN DAISY

(Coreopsis)

. Grandiflora........ Yellow.... Summer......24 in. 24 in.

(Rudbeckia) ....... Double............ Yellow....July-Aug.....3-5 ft. 25 in.

GRASS, ORNA-

MENTAL....... Eulalia Japonica . . ...............5-7 ft. $36 \mathrm{in.}$

HOLLYHOCKS.... Double............ Mixed......July-Aug......

MOSS PINK

(Phlox Subulata)..Alba............. White... April-May....2-6 in. 6 in.

\section{PAINTED DAISY}

(Pyrethrum) .... Hybridum. ...... Mixed....June-Oct.....18 in, 10 in.

PERENNIAL SWEETT PEA

(Lathyrus

Latifolius) ......Albus...........White... \} July-Aug. ....4-8 ft. $6 \mathrm{ft}$.

PHLOX PANICULATA

Splendens...........Pink....

$\star$ PHLOX PANICULATA

Miss Lingard...... White.........

Mrs. Chas Dorr . Lavender......

Richard Wallace white crimson eye Sum....24 in. 15 in.

Rheinlander, Salmon, claret eye....

Rijnstroom, bright crimson pink...

Jules Sandeau, Watermelon pink...

Thor, salmon pink.......... ) Summer......15 in. $12 \mathrm{in.}$

Beacon, cherry red............

PINKS

(Dianthus

Plumarius).....Clove Pinks.........Mixed..... Summer......10 in. 12 in.

PLAINTAIN LILY

(Funkia) ........... Caerulae.......... Blue.....July........24 in. 15 in.

SEDUM

(Stonecrop) ....... Spectabile.........Pink......Aug.-Sept....12 in. $12 \mathrm{in.}$

SHASTA DAISY

Stolonifera....Purplish Pink. .July-Aug.....6 in. 12 in.

(Chrysan themum

Maximum) .....Alaska..........White.....July-Aug....24 in. 18 in.

SWEET WILLIAM

(Dianthus

Barbatus) ......Mixed............ Various....May-June....18 in. 12 in

*WHITE ROCKCRESS

(A rabis Alpina)

*YELLOW DAY LILY

(Hemerocallis) ....Flava............Yellow....June ........30 in. 12-18 in.

SPIREA ASTILBE-Gladstone (white), America (pink); blooms June and July

Grows about 18 inches high on erect stalks, plant about 2 feet apart. By Mail

Postpaid, per each, 75c; per $3, \$ 1.75$; per $12, \$ 6.00$.

Varieties underlined can be planted in the fall. 


\section{EVERGREENS-Prices F. O. B. Neosho.}

The following prices are on good specimens, fresh dug with ball of earth wrapped in burlap, f. 0 . b. Neosho. There is no guarantee of grow th since that depends upon proper care on the part of the customer.

Directions-Submerge the earth ball in water 30 minutes in a shady place out of the wind. Set the tree slightly deeper than it stood in the nursery. Leave the burlap around the earth ball, but cut the strings after placing the tree in position. Tramp good dirt firmly around the tree. When the space around the ball is nearly filled, pour in several pails of water. Then fill in with loose dirt. Don't put any fertilizer in the holes. Loose straw or well rotted leaves spread around the tree make a good fertilizer and help protect against winter injury. Evergreens should be well watered during the summer and also in late fall.

The American Arborvi tae (Thuja Occidentalis) are the hardiest types from Kansas City north. The Chinese Arborvitae (Biota Orientalis) are more satisfactory for southern planting where they have to withstand the hot summer suns and protracted drought. Both types do well in the intermediate section, i. e., within 100 miles of Neosho.

AMERICAN ARBORVITAE $\ldots \ldots \ldots \ldots \ldots \ldots \ldots \ldots .2-3 \mathrm{ft}$

(Thuja Occidentalis)

Deep green, grows bushy at bottom, tapering towards top.

AMERICAN GLOBE ARBORVITAE

(Thuja Occidentalis Globosa)

15-18 in spread $\quad 3.00$

Deep green, natural globe shaped. $18-24$ in. spread $\quad 5.00$

Needs full sun. Narrow pyramidal form. $\quad 3-31 / 2 \mathrm{ft}$

$31 / 2-4 \mathrm{ft}$.

BAKER'S PYRAMIDAL ARBORVITAE

$2-21 / 2 \mathrm{ft}$.

(Biota Orientalis Pyramidalis)

$3-4 \mathrm{ft}$. $\quad 6.50$

$4-5 \mathrm{ft}$. $\quad 9.00$

$5-6 \mathrm{ft}$.

$\begin{array}{cr}\text { BERCKMAN'S GOLDEN ARBORVITAE............ } \\ \text { (Biota Orientalis Aurea Nana) } \\ 2-21 / 2 \mathrm{ft} . & 7.00 \\ 21 / 2-3 \mathrm{ft} . & 9.00\end{array}$

$\begin{array}{ccr}\text { (Biota Orientalis Aurea Nana) } & 21 / 2-3 \mathrm{ft} . & \mathbf{9 . 0 0} \\ \text { Rich golden. Compact globular form. } & 3-31 / 2 \mathrm{ft} . & \mathbf{1 2 . 0 0}\end{array}$

BONITA ARBORVITAE, Locke's Dwarf Globe....... \{21/2-3 ft. \} 6.00

(Bonite Arborvitae).

Rich green. Unrivalled in color and perfection of form.

COMMON CHINESE ARBORVITAE .............. $3-4 \mathrm{ft}$.

(Biota Orientalis)

Dark green, varying in shape and color as they are $5-6 \mathrm{ft}$.

grown from seed.

$6-7 \mathrm{ft}$.

$2-2 \frac{1}{2} \mathrm{ft}$. $\quad 4.50$

COMPACT CHINESE ARBORVITAE

(Biota Orientalis Compacta)

Dark green. Compact. Dwarf type.

$21 / 2-3 \mathrm{ft}$. $\quad 5.50$

MAYHEW'S GOLDEN ARBORVITAE...

(Biota Orientalis)

$3-4 \mathrm{ft}$.

4.50

6.00

7.00

4.50
5.50

7.50

entalis) $2-21 / 2 \mathrm{ft}$.

$3-31 / 2 \mathrm{ft}$.

ROSEDALE ARBORVITAE ................... $18-24$ in.

Bluish in winter. Feathery foliage. Compact. $\quad 2-21 / 2 \mathrm{ft}$.

( 3.00

4.00

5.00
6.00

3.00

4.00

2.50

$\begin{array}{lrl}\text { Similar to Rosedale, but darker and less compact. } & 2-21 / 2 \mathrm{ft} . & 3.50 \\ & 21 / 2-3 \mathrm{ft} . & 4.50 \\ 3-31 / 2 \mathrm{ft} . & 5.00\end{array}$

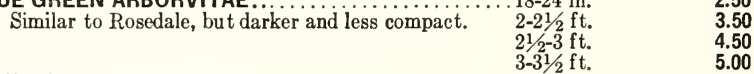

JUNIPERS

5.00

6.00

8.00

5.00

6.50

9.00

9.00
12.00

6.00

$\begin{array}{lll}\text { Dark green. Upright growing. Endures shade. } & 21 / 2-3 \mathrm{ft} & \mathbf{7 . 0 0} \\ & 3-31 / 2 \mathrm{ft} . & \mathbf{8 . 0 0}\end{array}$

$\begin{array}{lll}\text { Dark green. Upright growing. Endures shade. } & 21 / 2-3 \mathrm{ft} & \mathbf{7 . 0 0} \\ & 3-31 / 2 \mathrm{ft} . & \mathbf{8 . 0 0}\end{array}$

SPRUCE (Picea)

Black Hill Spruce ( 30 to $40 \mathrm{ft}$.) . . . . . . . . . $\quad 6.00$

(Picea Albertiana) Bluish-green. Compact grower. $3-31 / 2 \mathrm{ft}$. $\quad 8.00$

Norway Spruce. . . . . . $\quad 2.50$

(Picea Excelsa)

Rapid growing, 80-100 ft. Dark green foliage. $\quad 21 / 2^{-3} \mathrm{ft} . \quad 4.00$

Colorado Blue Spruce....................... $\begin{array}{r}3-4 \mathrm{ft} . \\ 18-24 \mathrm{in.}\end{array} \quad \begin{array}{r}6.00 \\ 9.00\end{array}$

Picea Pungens Glauca)

$21 / 2-3 \mathrm{ft}$. $\quad 18.00$

$3-31 / 2 \mathrm{ft}$. $\quad 24.00$

PINE (Pinus)

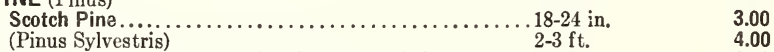

Rapid growing, 25 to $30 \mathrm{ft}$. Pale green foliage. $\quad 3-4 \mathrm{ft}$. $\quad 6.00$

Mugho Pine $(21 / 2-3 \mathrm{ft}$.) .........................

(Pinus Montana Mughus) Dark green, dwarf compact. 18-24 in. $\quad 7.50$

RETINOSPORA ANDELY....................18-24 in. $\quad 3.75$

Bluish-green. Dense compact, round headed, low $\quad 2-2 \frac{1}{2} \mathrm{ft}$. $\quad 4.50$ growing.

RETINOSPORA PLUMOSA.

$.2-3 \mathrm{ft}$.

5.00

Light green, dense, cone shaped.
RETINOSPORA PLUMOSA AUREA ................2-3 ft.

Golden yellow.

JAPANESE YEW (Taxus Cuspidata) ...............15-18 in.

Dark green, bushy type. Endures shade. $18-24$ in. 


\section{APPLE AND CRABAPPLE}

Prices F. O. B. Neosho, by Express or Freight.

Two-Year Branched.

Diameter

$\begin{array}{ll}\text { Height } & \text { Each } \\ 5 \text { Rate } & \end{array}$

Rate

$\$ 0.60$

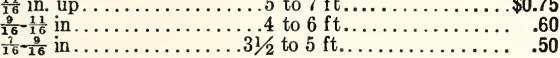

APPLE TABLE

Varieties marked ${ }^{*}$ are adapted only to northern climate.

Under "Size," M-Medium; M-L-Medium to Large; L-Large; V-L-Very Large.

Under "Quality," F-Fair; G-Good; V-G-Very Good; B-Best.

Under "Use," C means for Cooking; D for Dessert.

Varieties Color Size Quality Use Keeps in

EAREY SUMMER:
Liveland Raspberry.....Red Striped .......................... D... Yellow Transparent....Y Yellow........................... D. . 2 Weeks MIDSUMMER:

Red Astrachan....... Red Striped........M. to L.. G to V. G..C. D. . . 3 Weeks Duchess of Oldenburg. Red Striped ................G to V. G..C..... 2 Weeks Wilson Red June..... Deep Crimson.....M. to L..V. G....... D. C... 3 Weeks Sweet Bough..........Greenish-Yellow ... M.......G. to V. G..C. D... 2 Weeks Early Harvest......... Pale Yellow.............. G. to V. G. .C. D. .. 1 Week LATE SUMMER:

Maiden Blush......... Yellow-Red Blush.M......G........C. D... 7 Weeks Ada Red............ Purplish-Red.....M....... V. G....... D. C... 3 Weeks Wealthy .............Red Striped.......... to L...G. to V. G. .D. C. . 5 Weeks FALL:

UMcIntosh*........... Bright Red .......M. to L..V. G. to B.. D..... 9 Weeks L Grimes Golden....... Golden Yellow....M. to L..B......... D. C. . 10 Weeks King David ......... Dark Red.................... D. C..12 Weeks Wagener*............Red and Yellow...M. to L..V. G. to B..D. C...8 8 Weeks

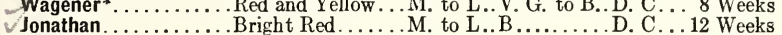
WINTER:

Delicious ...........Red Striped ......L...................10 Weeks

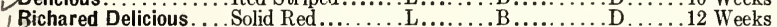
R. I. Greening*....... Green and Yellow.L................. C...12 Weeks Winter Banana......... Yellow and Red...M. to L..G. to V. G..D. .... 10 Weeks Spitzenburg*......... Yellow and Red...M. to L... B ......... D. C...12 Weeks Black Ben ......... Bright Red.......... to L..F..............14 Weeks Ben Davis ............. Red Striped .......... to L..F ................ Weeks Rome Beauty.........Red Striped........... to L..G........... C. . . 14 Weeks Baldwin*.............. Bright Red........... to L..G.......... D. C...14 Weeks Northern Spy* ...... Red Striped....................... D. C . .14 Weeks Winesap.............. Dark Red................ to V. G..D. C...14 Weeks Stayman...............Red Striped.................. to V. G..D. C...14 Weeks Senator (Oliver) ........ Dark Red..............................12 Weeks Mam. Black Twig..... Dark Red. ........................ D. C...16 Weeks Paragon ... Dark Red........M. to L..G. to V. G..D. C...16 Weeks York Imperial .........Red Striped..............G. to V. G..D. C...16 Weeks Willow Twig........... Green and Red.....M. to L..F. to G....C. D...16 Weeks Champion (Collins) ....Red and Yellow ...M....................16 Weeks Yellow Newtown ...... Yellow ............ to L.. B ......... D. C...18 Weeks CRABAPPLES: These varieties ripen about two weeks apart.

Florence............Red Over Yellow. M..................2 Weeks Excelsior.............. Red and Yellow... V. L .....G. to V. G..C. D... 2 Weeks Hyslop..............Red Over Yellow..M. to L..G............. 6 Weeks Whitney............Red Over Yellow..L........G. to V. G..C. D... 2 Weeks

TRIPLE THE LIFE OF YOUR GRIMES TREES by planting our double-worked trees. The Grimes part is budded about 18 inches from the ground on a hardy variety not susceptible to collar-rot. These Double-worked Grimes have roots and stock that are two seasons growth.

\section{RICHARED (Trade Marked) DELICIOUS}

This strain of common Delicious colors up a solid red two or three weeks earlier but is identical in flavor, size, shape and texture. The Richared originated near Wenatchee, Washington, in the orchard planted in 1910 of G. T. Richardson. Trees of the third generation grown from this original tree have borne fruit true-to-strain.

Wherever the common Delicious thrives this Richared will prove more profit able because it can be picked and marketed two weeks earlier with solid red color. Windfalls are avoided and the keeping quality is better.

Purchasers are required to sign an agreement not to sell or give away or dispose of any buds or scions from these trees.

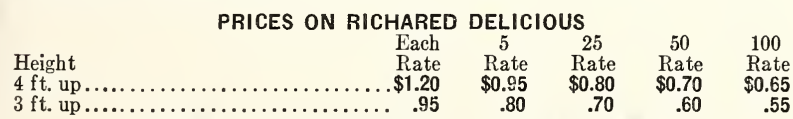

\section{DEMONSTRATION ORCHARDS}

Apple Trees-

2 yr., branched, $\frac{9}{16}$ in. diameter, 4 to $6 \mathrm{ft}$.

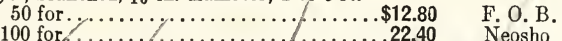

Your choice of Delicious, Wuchess, Grimes Golden, Jonathan, Stayman, Wealthy, Winesap, York Imperial.

Peach Trees- $\quad \frac{5}{16}$ to $\frac{7}{16}$ in. diameter, $3 \mathrm{ft}$. and up.

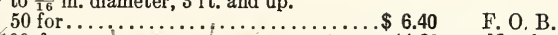

100 for ......................11.20 Neosho

Your choice of Belle of Georgia, Elberta, Greensboro, J. H. Hale, Late Elberta, Mayflower, Rochester. 


\section{PEACH}

Prices F. O. B. Neosho, by

Express or Freight.

Diameter Height Each Rate

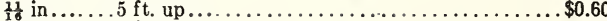

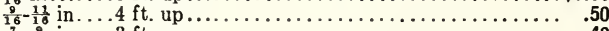

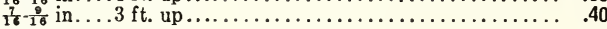

Rate Rate

$\$ 0.50 \$ 0.40$

Varieties in Seasons

Flesh

Size Quality

Days

Ahead of
Elberta

VERY EARLY:

Mayflower............White-Semi-cling....Medium .....Fair...... 50

EARLY:

Greensboro*.........White-Semi-free....Medium.....Fair....... 40

Arp (Beauty) ............. Yellow-Semi-cling ... Medium ...... Good......... 38

Erose (Early Rose) ...... Red \& White-Cling..Medium...... Very Good... 35

Carman*............ White-Semi-free.... Large....... Good ........ 24

MIDSEASON:

Alton............. White-Semi-free.... Large...... Good....... 18

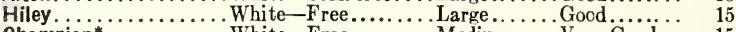

Champion*.............White-Free............. Medium..... Very Good... 15

Illinois.............White-Free......... Large...... Very Good... 15

Rochester........... Yellow-Free........Large....... Very Good... 15

Belle (of Georgia)* ..... White-Free........... Large........ Very Good... 8

J. H. Hale ............ Yellow-F ree............ Largest...... Very Good...

Early Elberta .......... Yellow-Free........ Very Large...Good....... 3

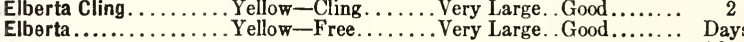

Elberta............. Yellow-Free........Very Large..Good........ $\begin{gathered}\text { Days } \\ \text { After }\end{gathered}$

Elberta

LATE:

Crosby**............ Yellow-Free........ Medium..... Very Good... 5

Late Crawford.......... Yellow-Free......... Very Large... Very Good.... 8

Late Elberta............ Yellow-Free.......... Large.......Good........ 20

VERY LATE:

Krummel.............Yellow-Free.......Large......Good....... 30

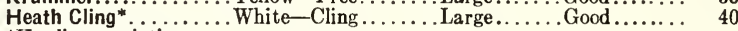

*Hardiest varieties.

\section{CHERRY}

Early Richmond (May Cherry), Montmorency, English Morello

These "Sour" Cherries ripen about a week -apart. The Sweets are more particular as to soil and climate and are sometimes self sterile, so a Black Tartarian should be planted with any other sweet variety.

Branched

Diameter

Height

Rach

$41 / 2 \mathrm{ft} \ldots \ldots \ldots \ldots \ldots \$ 1.00$

5
Rate
$\$ 0.85$

25

$\frac{11}{10}$ in......

$\frac{9}{16}-18$ in ..................... 3 ft. up........... $.75 \quad .65 \quad .55$

$\$ 0.75$

SWFET CHERRY Black Tartarian, Bing,Gov.Wood, Windsor, Napoleon (Royal Ann).

\begin{tabular}{|c|c|}
\hline & Heigh t \\
\hline
\end{tabular}

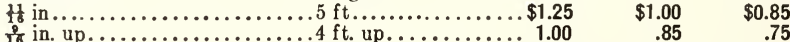

\section{PEAR - (Standard) Anjou, Bartlett, Flemish, Garber, Kieffer, Lincoln,} Seckel, Winter Nelis.

Pears are the highest quality fruits, should be picked before ripe. Lincoln, late July-Bartlatt, mid August-Flemish, mid August-Garber, early SeptemberAnjou, mid September-Duchess, late September-Seckel, late September-Kieffer, late September-Winter Nelis, early October.
Two-Year Branched.
Diameter
Height
Each
5
Rate
25
$\frac{11}{18}$ in.......
$.41 / 2 \mathrm{ft}$. up.
$\$ 0.85$
Rate
$\$ 0.75$
5
Rate
Rate
$\$ 0.50$
$\$ 0.65$
$\begin{array}{ll}\text { Ene-Year-Whips. } & \text { Each } \\ \text { Rate }\end{array}$

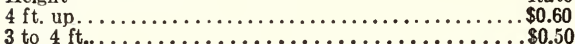

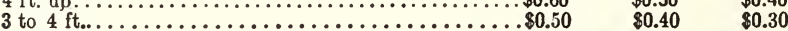

\section{PEAR-(Dwarf)}

Anjou, (Bartlett, Seckel, LDuchess d'Angouleme.

Diameter

Height

Each

Rate

Rate

$5 / 8$ in. up

$3 \mathrm{ft}$. up

$\$ 0.75$

QUINCE

Diameter

$5 / 8$ in .

Height

Each

Rate

5

$\$ 0.80$

\section{GRAPES}

Two-Year, No. 1.

Concord (Black) $\ldots \ldots \ldots \ldots \ldots \ldots \ldots \ldots \ldots \ldots \ldots \ldots \ldots \ldots \ldots \ldots \ldots \ldots \ldots$
Moore Early (Black) $\ldots \ldots \ldots \ldots \ldots \ldots \ldots \ldots$

$3 \mathrm{ft}$. up..................\$1.00

Agawam (Red)

Catawba (Red) $\ldots \ldots \ldots$

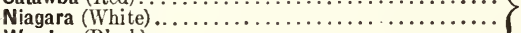

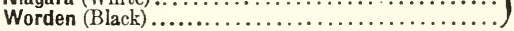

Campbell (Black). 


\section{PLUM}

Diameter

$\frac{11}{16}$ in........ Height Each

Rate

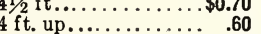

$\frac{16}{16}-18$ in

$\frac{9}{16}$ in $\ldots \ldots \ldots \ldots \ldots \ldots \ldots \ldots \ldots .3 \mathrm{ft}$. up............. 50

JAPANESE VARIETIES:

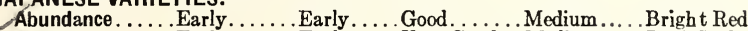

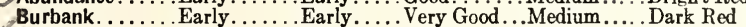

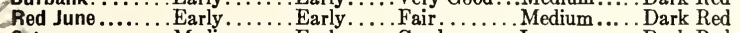

Satsuma ............ Medium ...... Early..... Good ........ Large....... Dark Red

NATIVE VÄRIËTIES:

tOmaha ........ Early . . . . . . . . . . . . Good . . . . . Large........ Light Red

Wild Goose....... Very Early.. Medium ... Fair......... Medium..... Bright Red

HYBRID VARÏ.... Ver

America........ Medium.....Medium... Medium... . Medium..... Golden Yel.

Endicott..... . . Early. . . . . . . . . . . . Good...... . Large . . . . . . Dark Red

(Mammoth Gold)

Wickson..........Medium.... . Medium...Good. ...... Very Large. . Dark Red

DOMESTICA VARIETIES:

Bradshaw.......Medium.... Medium...Good ........ Medium ... . Purplish-Red

Green Gage. ... . Medium .... . Medium .... Best... ...... Medium.... . Yellow (Reine Claude)

LItalian Prune... Late...... Late..... Good...... . Large...... Dark Blue

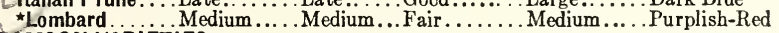
DAMSON VARIETIES:

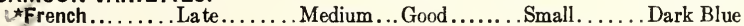
*Shropshire...... Late........ Late...... Fair. ....... Small . . . . . . Dark Blue HANSEN HYBRIDS:

$\star$ Hanska. ....... Very Early. . Medium...Very Good ...Large........ Bright Red $\star$ Opata ......... . Very Early. . Medium... Good ........ Large........ Dark Red *Sapa......... Very Early. Medium... Good ........ Medium .... . Dark Purple «Waneta........ Very Early. . Medium... Very Good ...Very Large. Red

The Italian Prune is a freestone. The others are cling or semi-clings.

$\star \star$ Very hardy.

CHERRY-PLUM, Compass, is very hardy, bears young. Fruit bright red, sweet, juicy.

\section{APRICOT-(Superb)}

has beautiful golden sweet fruit. Tree is hardy. Very good quality.

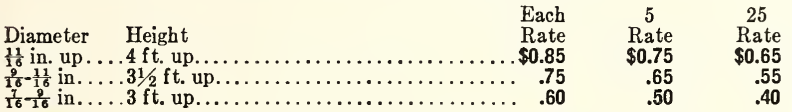

SIRA WBERRES No. 1 plants grown in new beds.

\section{STANDARD VARIETIES: Per 25 Per 50 Per 100 Per 250}

Dunlap (Midseason)...............

EVERBEARING V̈̈RIETIES:

$\begin{array}{lllll}\text { Champion............... } & & & \\ \text { Progressive }\end{array}$

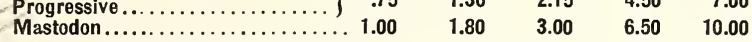

BIACIKBERRTES $\quad \begin{array}{ccc}\text { Per } & \text { Per } & \text { Per } \\ 10 & 25 & 100\end{array}$

Eldorado..............................\$0.75 $\$ 1.50 \quad \$ 5.00$

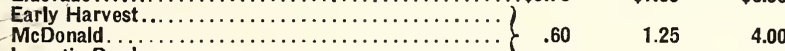

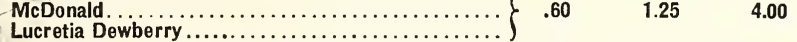

RASPBERRIES Per $\quad$ Per $\quad$ Per

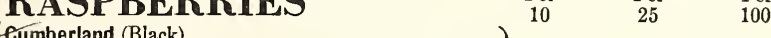

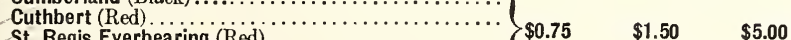

St. Regis Everbearing (Red) ................. $\$ 0.75 \quad \$ 1.50 \quad \$ 5.00$

Latham (Red) $\ldots . . .001 .00$

Latham (Redpath or Minnesota No. 4), bright red, extra large, ripens early_and over a long season, very productive and hardy.

GOOSERERRIES Two-Year, No. 1 Plants.

Downing............................. for $\$ 0.75 \quad 12$ for $\$ 2.50$

Oregon Champion............................. for $1.00 \quad 12$ for 3.25

CURRANTS Two-Year, No. 1 Plants.

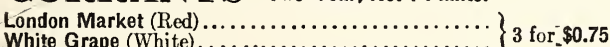

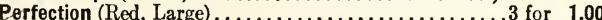

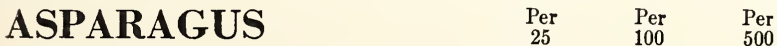

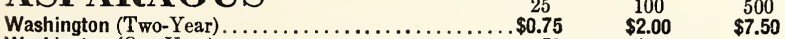

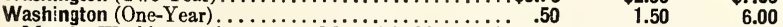

Martha Washington (two-year), Mary Washington (one-year). These are the best strains, resistant to rust.

RHUBARB selected crowns, 3 tor 80.50 


\section{MAIL SIZES—Postpaid}

This list shows sizes and quantities we can send by prepaid parcel post in the United States east of the Rocky Mountains. You will be agreeably surprised with the quality of stock you receive.

SHRUBS-Prices for 3 and for 10

ALTHEA $\ldots \ldots \ldots \ldots \ldots \ldots \ldots \ldots .18-24$ in .............. $\$ 1.10$

Per 10

ALMOND, DOUBLE FLOW $\quad \begin{aligned} & 12-18 \mathrm{in} \ldots \ldots \ldots \cdots \cdots \cdots \\ & .90\end{aligned}$

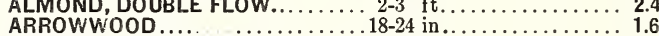

BARBERRY, JAPÄNESE. .............

BARBERRY, RED LEAVED . . . . . . $12-15$ in. . . . . . . . . . 2.10

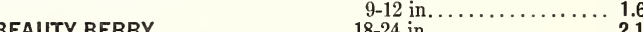

$18-24$ in $\ldots \cdots \cdots \cdots \cdots \cdots+1.62$

BUTTERFLY BUSH................... Medium...................

CORALBERRY $\ldots \ldots \ldots \ldots \ldots \ldots .18-24$ in $\ldots \ldots \ldots \ldots \ldots 1.05$

DESMODIUM . . .

DEUTZIA GRACILIS (White).......18-24 in $\ldots \ldots \ldots 1.65$

$12-18$ in $\ldots \ldots \ldots \ldots \ldots \ldots \ldots, 1.32$

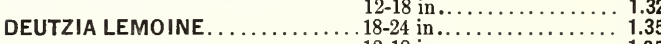

$12-18$ in ........

DEUTZIA, PRIDE OF ROCHESTER, $2-3 \mathrm{ft} \ldots \ldots \ldots \ldots \ldots \ldots$

$18-24$ in.................... 1.05

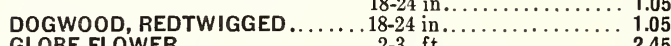

GLOBE FLOWER $\ldots \ldots \ldots \ldots \ldots \ldots \ldots, 2-3 \mathrm{ft} \ldots \ldots \ldots \ldots \ldots \ldots \ldots, 2.45$

GOLDEN BELL

$18-24$ in............... 2.10

Border....................

$2-3$ ft.............. 1.40

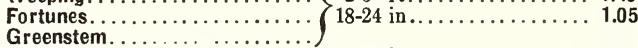

HIGH BUSH CRANBERRY......... 18-24 in ............... 1.95

HONEYSUCKLE

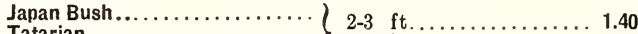

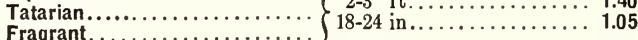

HYDRANGEA A. G................... 18-24 in ............. 1.35

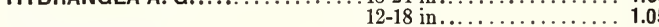

HYDRANGEA P. G.............. 18-24 in ...............

$12-18$ in $\ldots \ldots \ldots \ldots \ldots \ldots \ldots, 1.35$

HYPERICUM . . . . . . . . . . . . . $18-24$ in................... 1.95

JAPAN QUINCE................. 18-24 in ............. 1.35

JETBEAD or KERRIA ........... 18-18 in in........... 1.05

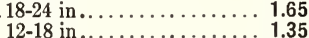

LILAC, BUDDED . ............ $2-3 \mathrm{ft} \ldots \ldots \ldots \ldots \ldots \ldots .4 .00$

$18-24$ in $\ldots \cdots \cdots \cdots \cdots \cdots+3.16$

LILAC, COMMON PURPLE....... 18-24 in ............... 1.20

$12-18$ in $\ldots \cdots \cdots \cdots \cdots \cdots \cdots \cdots \cdots+.90$

PERSIAN PURPLE. ............. $18-24$ in.................. 1.50

$12-18$ in ................ 1.35

MOCK ORANGE

Coronarius................ 2-3 ft........... 1.40

$18-24$ in.....................

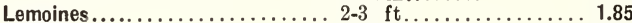

$2-3$ ft $\ldots \cdots \cdots \cdots \cdots \cdots \cdots \cdot 1.85$

Golden................... $18-24$ in .................... 2.90

Virginalis..............................

PRIVET, REGELS ................. 18-24 in ............... .90

SNOWBALL .............. 18-24 in $\ldots 1.80$

$12-18$ in ....................

SNOWBERRY............... $18-24$ in $\ldots \ldots \ldots \ldots \ldots \ldots \ldots, 1.05$

SPIREA

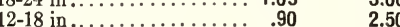

Sp. Thunbergi .............. 18-24 in............. 1.35

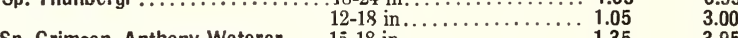

Sp. Crimson, Anthony Waterer. . $15-18$ in ......................

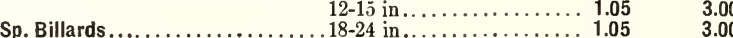

$\begin{array}{rrr}18-24 \text { in } \ldots \ldots \ldots \ldots \ldots \ldots & 1.05 \\ 12-18 \text { in } & 3.00 \\ 2.50\end{array}$

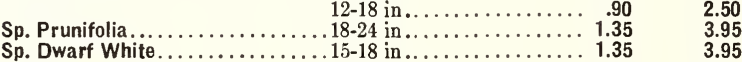

$12-15$ in ............... $1.05 \quad 3.00$

Sp. Dwarf Rosea ...............18-24 in................ $1.65 \quad 4.86$

Sp. Froebel's....................

Sp. Van Houttei $\quad 12-18 \mathrm{in} \ldots \ldots \ldots \ldots \ldots \ldots \ldots+1.05$

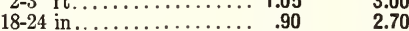

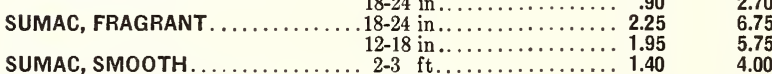

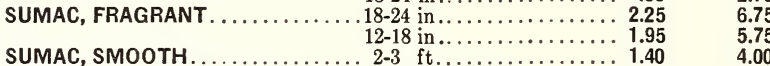

TAMARISK, AFRICANA . . . . . . . .

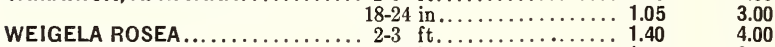

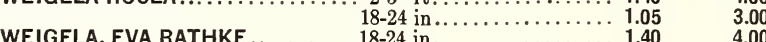

HEDGE-Prices for 10 , for 30 , for 100

\begin{tabular}{|c|c|c|c|}
\hline CALIFORNIA PRIVET.. & $\begin{array}{r}\text { Per } 10 \\
\ldots \ldots 1.20\end{array}$ & $\begin{array}{c}\text { Per 30 } \\
\$ 2.86\end{array}$ & $\begin{array}{r}\text { Per } 100 \\
\$ 7.00\end{array}$ \\
\hline & $12-18$ in... & 2.16 & $\begin{array}{r}\$ 7.00 \\
4.76\end{array}$ \\
\hline AMUR PRIVET. & $.18-24$ in.... & 4.66 & 13.00 \\
\hline & $12-18$ in... & 3.36 & 8.76 \\
\hline BARBERRY, JAPANESE & $15-18$ in. . & 5.00 & \\
\hline SPIREA, VAN HOUTTEI & 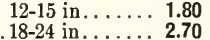 & $\begin{array}{l}4.66 \\
6.46\end{array}$ & 14.00 \\
\hline & $12-18$ in...... 1.66 & 3.96 & 11.76 \\
\hline
\end{tabular}




\section{MAIL SIZES-Postpaid.}

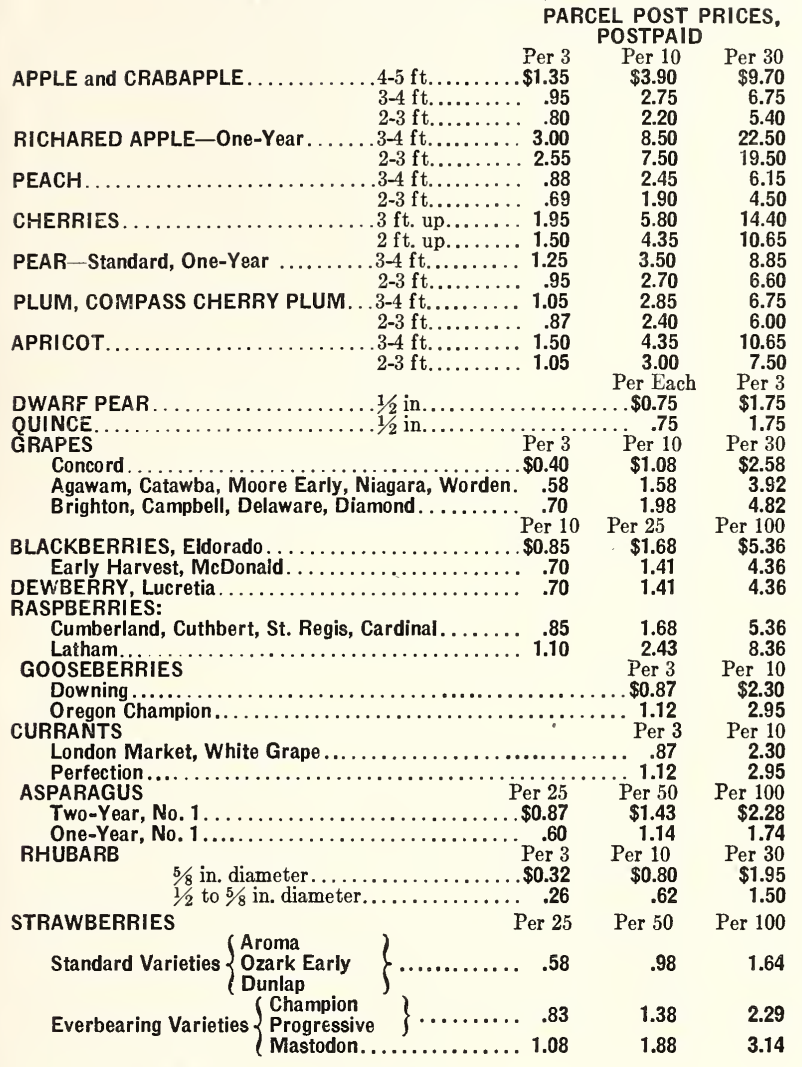

\section{FALL BULBS-Postpaid.}

Plant in September, October and November

\section{TULIPS}

DARWIN-Collection, named varieties our selection, good varieties, 75c for 12 , $\$ 1.25$ for 25 , and $\$ 4.50$ for 100 .

DARWIN-Select Varieties.

Per 6 Per 12 Per 25 Per 100

Clara Butt-Apple blossom pink, late.

Bartigon-Dazzling crimson, early. Best

for indoor forcing.

Mrs. Potter Palmer-Bright purplish violet, late.

Rev. H.'Ewbank-Heliotrope-lilac, early.

Zulu-Purple-black, late.

EARLY SINGLE TULIPS-Collection $\quad \begin{array}{lllll} & & 40 & .75 & 1.25\end{array}$

Our selection of mixed varieties.

$\begin{array}{llllll}\text { Chrysolora -Golden Yellow.................. } & .75 & 1.40 & 2.50 & 9.00\end{array}$

Cramoise Brilliant-Crimson............... $\quad .50 \quad .85 \quad 1.50 \quad 5.50$

$\begin{array}{lllll}\text { Kaiser Kroom-Red and yellow.............. } & .85 & \mathbf{1 . 5 0} & \mathbf{2 . 7 5} & \mathbf{1 0 . 0 0}\end{array}$

EARLY DOUBLE TULIPS-Collection..............

Our selection of varieties.

Lucretia-Deep pink................. .60

$\begin{array}{lll}1.00 & 1.75 & 6.00\end{array}$

\section{HYACINTHS}

(Bulbs 15 to $16 \mathrm{c.m}$.)

Mixed colors, our selection

L'Innocence-Pure white
La Victoire-Rosy red.

Ka Victoire-Rosy red.

Gertrude-Rosy pink.

\section{NARCISSUS}

Yellow Trumpet.

for 75 c, 12 for $\$ 2.50,25$ for $\$ 4.50$

Paper White.....................................

\section{LILIES}

Gold Banded Lily-(Lilium Aura tum)

Lily of the Valley-(Ccnvallaria Majalis)................ Clumps 70c each, 3 for $\$ 1.65$

Niadonna Lily-(Lilium Candidum).......................... each, 3 for $\$ 1.60$

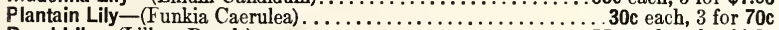

Regel Lily-(Lilium Regale).........................55c each, 3 for $\$ 1.35$

W hite Day Lily-(Funkia Subcorda ta) . . . . . . . . .

Yellow Day Lily-(Hemerocallis Flava)....................... each, 3 for $70 \mathrm{c}$ 


\section{GET ACQUAINTED OFFERS}

People who try our stock and service send us repeat orders and recommend us to their friends. That is one good reason for these offers. We also wish to share our good fortune in securing exceptionally good results in production.

These Specials are subject to prior sale and cash with order.

\section{HOME FRUIT GARDEN COLLECTIONS}

\section{F. O. B. Neosho-By Express or Freight. Cash With Order.}

\begin{tabular}{|c|}
\hline 12 Peach Trees-1 Yr.... \\
\hline 9 Cherry Trees... \\
\hline 8 Plum Trees $-4 \mathrm{ft}$ up, 1 Apricot \\
\hline
\end{tabular}

(We will select varieties of fruit trees adapted to your district, the best in quality and dependabiilty.)

18 Grape Vines -2 Yr., No. $1 \ldots \ldots \ldots \ldots \ldots \ldots \ldots \ldots \ldots \ldots \ldots \ldots \ldots \ldots . . \ldots 0$

(2 red, 2 white, 4 Moore Early, 10 Concord.)

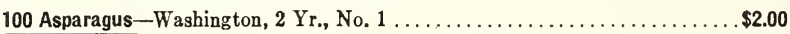

125 Strawberries-Everbearing ............................... $\$ 2.90$

(100 Progressive, 25 Mastodon.)

100 Raspberry.

(25 St. Regis, 25 Latham, 50 Cumberland.)

On order for all the above trees and plants amounting to $\$ 31.20$ we will pay transportation charges. Shipment will be made at proper tlme for planting.

THESE TREES AND PLANTS TAKE A SPACE 128 BY 117 FT.

Row $A-5$ apple and 4 peach al ternating, $16 \mathrm{ft}$. apart.

Row B-16 ft. from A-9 cherry, $16 \mathrm{ft}$. apart.

Row C-16 ft. from B-Same as A.

Row D-16 ft. from C-8 Plum, 1 Apricot, $16 \mathrm{ft}$. apart.

Row E-16 ft. from D-Same as A.

Row F-25 ft. from E-18 Grape vines, $8 \mathrm{ft}$. apart.

Row G-10 ft. from F-100 Asparagus, 15 in. apart.

Row $\mathrm{H}-6 \mathrm{ft}$. from $\mathrm{G}-125$ Strawberries, $1 \mathrm{ft}$. apart.

Row I-6 ft. from $\mathrm{H}-50$ Raspberry, $2 \frac{1}{2} \mathrm{ft}$. apart.

Row $\mathrm{J}-6 \mathrm{ft}$. from $\mathrm{I}-50$ Raspberry, $2 \frac{1}{2} \mathrm{ft}$. apart.

\section{DOLLAR OFFERS---POSTPAID}

4 Apple Trees $-\frac{5}{16}$ to $\frac{7}{16}$ in. diameter, $3 \mathrm{ft}$. and up-for......... $\$ 1.00$ Postpaid Your choice of Yellow Transparent, Duchess, Wilson Red, Wealthy, Jonathan, Delicious, Stayman, Winesap.

4 Peach Trees $-\frac{8}{16}$ to $\frac{7}{16}$ in. diameter, $3 \mathrm{ft}$. and up - for.........\$1.00 Postpaid

Your choice of Mayflower, Greensboro, Early Rose, Champion, Rochester, Belle of Georgia, Elberta, Late Crawford.

6 Peach Trees $-2-3 \mathrm{ft}$. Choice of above varieties - for .......... \$1.00 Postpaid

4 Plum Trees-2-3 ft. yearlings - for .................... $\$ 1.00$ Postpaid

Your choice of Endicott (Mammoth Gold), Green Gage, Italian Prune, Red June, Wild Goose, Waneta.

3 Cherry Trees $-2-3 \mathrm{ft}$. yearlings - for.................. $\$ 1.00$ Postpaid

(1 Early Richmond, 1 Montmorency, 1 English Morello.)

12 St. Regis Raspberry (Everbearing, red) -for ..............\$1.00 Postpaid

1 Bechtel Double Flowering Crab-2-3 ft.-(See back cover) -for . . . \$1.00 Postpaid

3 Shrubs for.................................\$1.00 Postpaid

Your choice of Japanese Barberry, Golden Bell, Japan Bush Honeysuckle, Japan Quince, Purple Lilac, Mock Orange, Spirea Billardi, Tamarix Africana, all 18-24 in., and Spirea Van Houttei 2-3 ft.

4 Shrubs for................................\$1.00 Postpaid Your choice of Purple Lilac, Spirea Billardi, Spirea Van Houttei, Spirea Froebeli, Japanese Barberry, all 12 to 18 in.

10 Grape VInes-2 yr., No. 1 (7 Concord and 3 Moore Early) -for. ...\$1.00 Postpaid

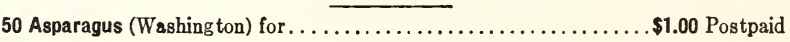

\section{ROSES}

1 white, 1 yellow, 2 red, 2 pink, \$3.65 Postpaid

Popular Everblooming varieties. The very best grade 2 yr. field grown. 


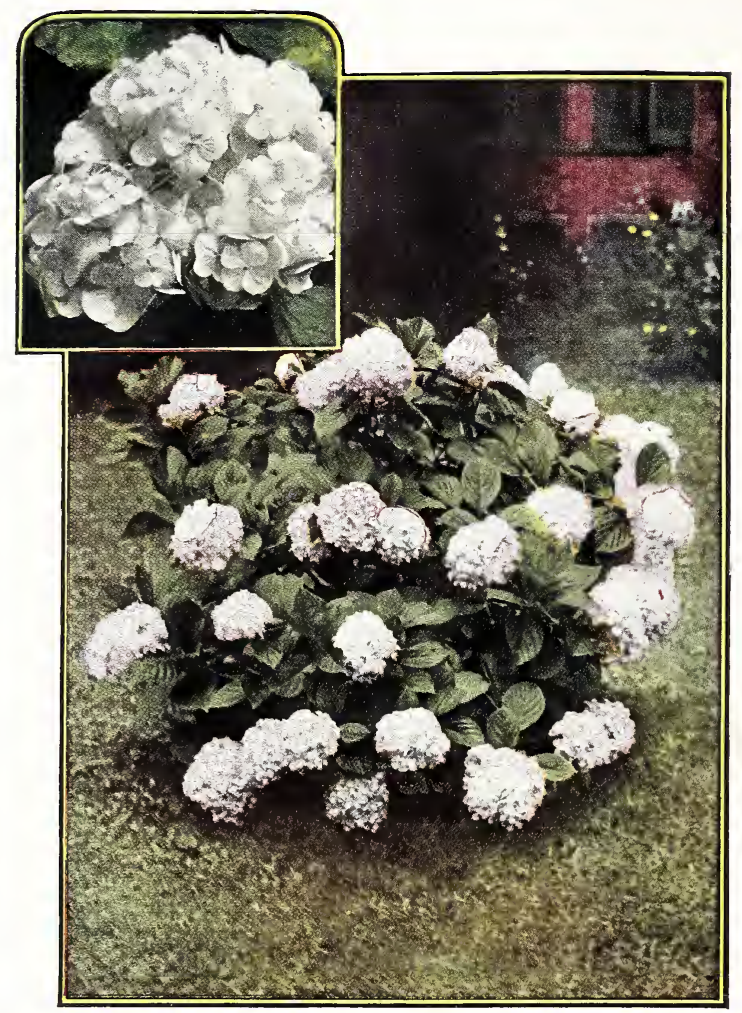

HYDRANGEA ARBORESCENS

Splendid for Shady Places. Blooms Aug.-Oct.

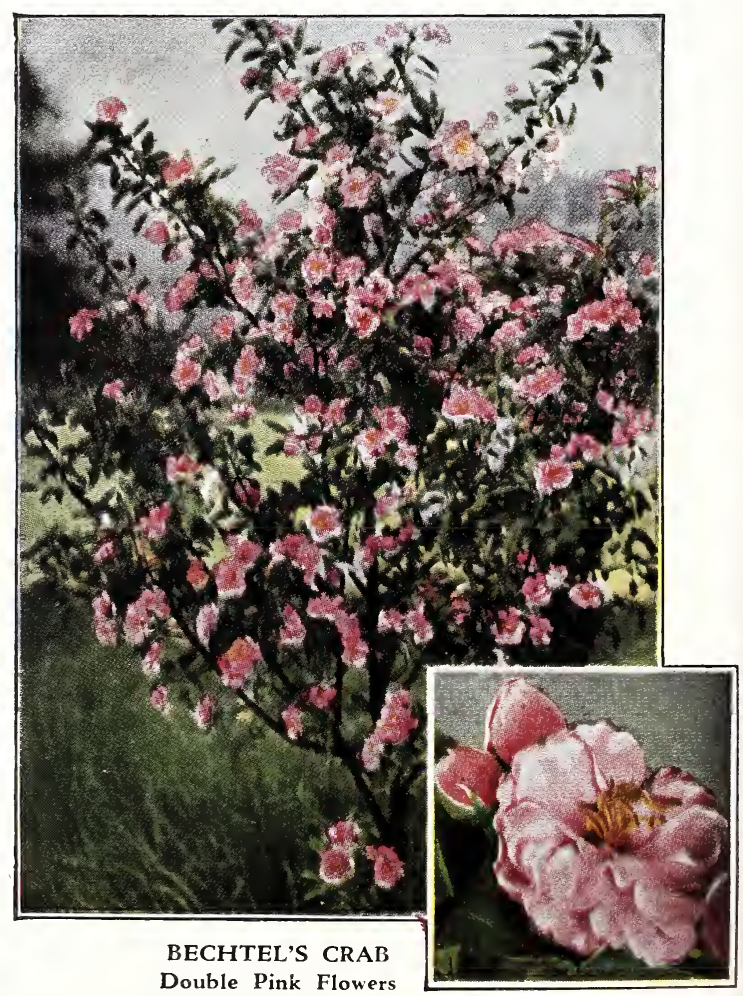

THE GENESEE PRESS, ROCHESTER, N Y 